\title{
A Methodology for Cable Damage Identification Based on Wave Decomposition
}

\author{
Songhan Zhang ${ }^{\mathrm{a}, \mathrm{b}, *}$, Ruili Shen ${ }^{\mathrm{b}}$, Kaoshan Dai $^{\mathrm{a}}, \mathrm{Lu} \mathrm{Wang}^{\mathrm{b}}$, Guido De Roeck $^{\mathrm{c}}$, Geert Lombaert ${ }^{\mathrm{c}}$ \\ ${ }^{a}$ Department of Civil Engineering, Sichuan University, China \\ ${ }^{b}$ School of Civil Engineering, Southwest Jiaotong University, China \\ ${ }^{c}$ Department of Civil Engineering, KU Leuven, Belgium
}

\begin{abstract}
Vibration-based damage identification has been widely studied in the field of structural health monitoring (SHM) for several decades. It is well known, however, that low-order modal parameters, being among the most frequently used, are not sensitive to local damage. A suitable methodology is therefore needed to extract such damage features from the dynamic response of structures. In the present work, local bending behavior of cables is studied for damage identification. First, the dynamic response of a cable is decomposed into evanescent wave and propagating wave components. It is proven that the contribution of the evanescent wave is spatially concentrated, and is sensitive to local damage. A signal transform is proposed next, which allows the estimation of the wave components from the measured cable response. The reflection coefficient of the evanescent wave (REW), which can be calculated from the estimated wave coefficients, depends only on the characteristics of the local discontinuity, and proves to be a robust indicator for local damage. The feasibility of the proposed methodology is studied by means of a simulated experiment, considering a cable model with two locally damaged parts. The results show that the intensity of REW is significantly higher near the damage locations, allowing damage localization. From the estimated REW near the damage locations, the damage levels can be estimated, showing the potential of this methodology for damage assessment of cable structures.
\end{abstract}

Keywords: cable; bending wave; wave decomposition; evanescent wave; signal transform; damage identification

\section{Introduction}

Cables are widely used in large-span engineering structures (e.g. cable-stayed bridges, suspension bridges and cableways), due to their high load capacity and low own weight [1. However, most of such structures are located in harsh environment with heavy traffic loading, and have inevitable corrosion 2 or fatigue 3 damage. As cables are critical parts, the development of such local damage might rapidly endanger the main structure. It is therefore important to detect and quantify local damage prior to the occurrence of a failure.

Cables usually consist of multiple wires protected in the PE pipe, and a large-span structure contains many such cables 4. The visual inspection of local damage requires the dissection of PE pipe, which is neither convenient nor realistic for long-term health monitoring [5]. The image-based technique developed from feature recognition has been applied for the automatic inspection of stay cables, but is only effective for damage at the surface 6 . New approaches such as thermography, impulse radar, pulse magnetic response are developed [7, which allow detecting internal damage by means of indirect measurements [8]. However, most of these techniques involve costly equipment and manual operation.

\footnotetext{
* Corresponding author

Email address: songhan.zhang@scu.edu.cn (Songhan Zhang)
} 
During the past decades, modal parameters, which can be identified from operational vibration data 9, became one of the most popular features for health monitoring of structures. Reductions in natural frequencies represent a decrease of structural stiffness, indicating possible local damage [10. A sudden increase in modal curvature indicates a reduction in local stiffness [11, revealing possible local damage [12. The above methodologies have been extensively applied in real cases 13 , but the following issues still remain to be solved:

First, lower-order modal parameters, characterizing global dynamic behavior, are insensitive to local damage. Especially for cables connected to a flexible structure, the changes in natural frequencies resulting from damage might not be a reliable or suitable indicator of local damage due to the redistribution of cable forces [14. Besides, measuring modal curvatures of cables requires a dense measurement [15], whereas in practice, only a few measurement points are available. Lastly, even when a decrease in natural frequency is detected, this may be due to change in temperature, degeneration of the connections to main structure, decrease in static loading besides local damage, resulting in an incorrect diagnosis.

With the development of distributed measurements by means of FBG sensors, modal-based damage identification, involving modal strains [16, has regained interest [17] as they are direct indicators of local bending stiffness 18. However, a physical contact between the sensor and the surface of the material is required, which is difficult for existing cables as the strands are usually protected inside.

An alternative to the modal-based damage identification involves wave propagation features, as a reflected wave is generated at a damage location [19]. Theoretically, only one or two sensors are sufficient to capture propagating waves, including the incident wave and reflected wave. From the travel time and the intensity of the reflected wave, local damage can be located and quantified 20. Due to this advantage, guidedwave-based methods have been studied and applied for damage detection of slender structures, such as pipes 21, rails 22, as well as multi-wire cables 23. Applications of guided waves mainly focused on the longitudinal waves, excited by a harmonic wave signal with a short wavelength [24. Such longitudinal guided waves, however, are complicated in a multi-wire cable due to the coupling between wires [25]. On the other hand, the longitudinal motion of wires can be disregarded for the transverse waves involving cable bending, as long as the wavelength is much larger than the dimensions of the cross section [26. It has been proven that the bending wave is sensitive as well to local damage 20. However, dispersion makes the wave signal distorted, and the reflected wave signal generated by a local damage is usually polluted by additional reflections from boundaries and multiple damages. This may complicate the signal interpretation, requiring a suitable method to extract damage sensitive features [27].

The analytical solution of the frequency-domain response of a cable is formed by a superposition of four terms, which represent evanescent wave and propagating wave components. Among them, the evanescent wave component is not only sensitive to local discontinuities but is also spatially concentrated, which allows damage identification even when present at multiple locations. In the present work, a signal transform is developed for decomposing the frequency-domain response of a cable into evanescent wave and propagating wave components. Based on this, the reflection coefficient of the evanescent wave (REW), which depends only on the characteristics of a local discontinuity of the cable, can be estimated, and is shown to be a suitable indicator for local damage.

The present work consists of three parts. First, the transverse motion of a cable with local damage is derived through a piecewise analytical solution. The sensitivity of the evanescent wave to local damage is studied. Next, the wave decomposition is implemented by means of the developed signal transform, allowing for the estimation of the coefficient of each wave component. The formula for the REW estimation is then derived. Finally, an experiment, simulated by a numerical model, is performed for a feasibility study of the proposed methodology. 


\section{Wave components of the uniform part of a cable}
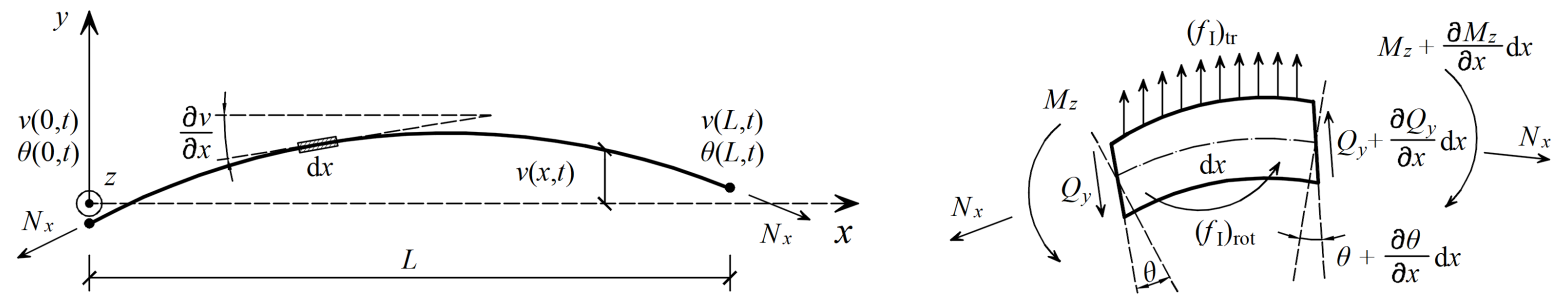

Figure 1: Model of a uniform cable without local damage

A cable is modeled as a Timoshenko beam with constant axial force $N_{\mathrm{x}}$ (Fig. 1). The following assumptions are made:

- the transverse displacement of the cable is much smaller than the total length;

- the cable sag under its own weight is disregarded;

- viscous damping with regard to transverse motion and shear deformation is assumed negligible;

- the orientation of the axial force remains tangent to the axis of the cable;

- the cable is assumed to be excited at its boundaries only.

In reference [20, the analytical solution of the dynamic response of the cable in the frequency domain is derived as:

$$
\begin{aligned}
& \hat{V}(x, s)=\tilde{C}_{1} \exp \left(k_{1} x\right)+\tilde{C}_{2} \exp \left(k_{2} x\right)+\tilde{C}_{3} \exp \left(k_{3} x\right)+\tilde{C}_{4} \exp \left(k_{4} x\right) \\
& \hat{\Theta}(x, s)=R_{1} \tilde{C}_{1} \exp \left(k_{1} x\right)+R_{2} \tilde{C}_{2} \exp \left(k_{2} x\right)+R_{3} \tilde{C}_{3} \exp \left(k_{3} x\right)+R_{4} \tilde{C}_{4} \exp \left(k_{4} x\right)
\end{aligned}
$$

where $\hat{V}(x, s)$ and $\hat{\Theta}(x, s)$ are the Laplace transforms of the transverse motion $v(x, t)$ and the rotation of cross section $\theta(x, t)$, respectively. The complex frequency is $s=\sigma+i \omega(i=\sqrt{-1}), \omega$ is the circular frequency. $\sigma$ is a real constant, representing a hypothetical exponential decay of the signal [28. The frequency-dependent constants $\tilde{C}_{j}(j=1,2,3,4)$ depend on the boundary conditions. $R_{j}(j=1,2,3,4)$ represent the ratio between the transverse motion and the rotation of the cross section in the frequency domain, given as [20]:

$$
R_{j}=\frac{\kappa G A k_{j}^{2}-N_{x} k_{j}^{2}+s^{2} \rho A}{\kappa G A k_{j}} \quad(j=1,2,3,4)
$$

where the wavenumbers $k_{j}(j=1,2,3,4)$ are given by the following dispersion relations [20]:

$$
\begin{aligned}
& k_{1}=-\sqrt{\frac{-\beta+\sqrt{\beta^{2}-4 \alpha \gamma}}{2 \alpha}} \\
& k_{2}=+\sqrt{\frac{-\beta+\sqrt{\beta^{2}-4 \alpha \gamma}}{2 \alpha}} \\
& k_{3}=-\sqrt{\frac{-\beta-\sqrt{\beta^{2}-4 \alpha \gamma}}{2 \alpha}} \\
& k_{4}=+\sqrt{\frac{-\beta-\sqrt{\beta^{2}-4 \alpha \gamma}}{2 \alpha}}
\end{aligned}
$$


where

$$
\begin{aligned}
\alpha & =E I\left(\kappa G A+N_{x}\right) \\
\beta & =-\kappa G A N_{x}-s^{2} E I \rho A-s^{2} \kappa G A \rho I-s^{2} \rho I N_{x} \\
\gamma & =s^{2} \rho A\left(\kappa G A+s^{2} \rho I\right)
\end{aligned}
$$

where $E$ and $G$ are the elastic modulus and the shear modulus of the material, $\kappa$ is the shear coefficient of cross section, $A$ and $I$ are the cross-section area and the moment of inertia, respectively, while $\rho$ is the density of material.

Suppose $\sigma=0$ for the frequency-domain analysis. From the dispersion relations given by Eq. (4 - 7), $\omega_{\mathrm{c}}=\sqrt{(\kappa G A) /(\rho I)}$ is defined as the cut-off frequency. When $\omega \leq \omega_{\mathrm{c}}$, the wavenumbers $k_{1}$ and $k_{2}$ are real, so $\tilde{C}_{1} \exp \left(k_{1} x\right)$ and $\tilde{C}_{2} \exp \left(k_{2} x\right)$ represent the evanescent non-propagating waves decaying exponentially along the positive and negative directions, respectively. The wavenumbers $k_{3}$ and $k_{4}$ are imaginary, $\operatorname{so} \tilde{C}_{3} \exp \left(k_{3} x\right)$ and $\tilde{C}_{4} \exp \left(k_{4} x\right)$ represent the propagating bending $(\mathrm{PB})$ waves along the positive and negative directions, respectively. When $\omega>\omega_{\mathrm{c}}$, all wavenumbers $k_{1}, k_{2}, k_{3}, k_{4}$ are imaginary. $\tilde{C}_{1} \exp \left(k_{1} x\right)+\tilde{C}_{2} \exp \left(k_{2} x\right)$ and $\tilde{C}_{3} \exp \left(k_{3} x\right)+\tilde{C}_{4} \exp \left(k_{4} x\right)$ represent propagating shear (PS) waves and bending (PB) waves, respectively.

\section{Wave reflection and transmission at a damage interface}

\subsection{Theoretical derivation}

In this part, the wave reflection and transmission at the interface between an undamaged and damaged part of a cable will be studied based on the analytical solution given by Eq. (1 - 22. The solution will be decomposed by considering each of the terms $\tilde{C}_{1} \exp \left(k_{1} x\right), \tilde{C}_{2} \exp \left(k_{2} x\right), \tilde{C}_{3} \exp \left(k_{3} x\right)$ and $\tilde{C}_{4} \exp \left(k_{4} x\right)$ separately. At the damage interface, a sudden change in cross section occurs (Fig. 2), and its effect on the above wave components will be studied. In order to exclude the effect of the physical boundaries, the cable is assumed infinitely long, modeled with absorbing boundaries at both sides.

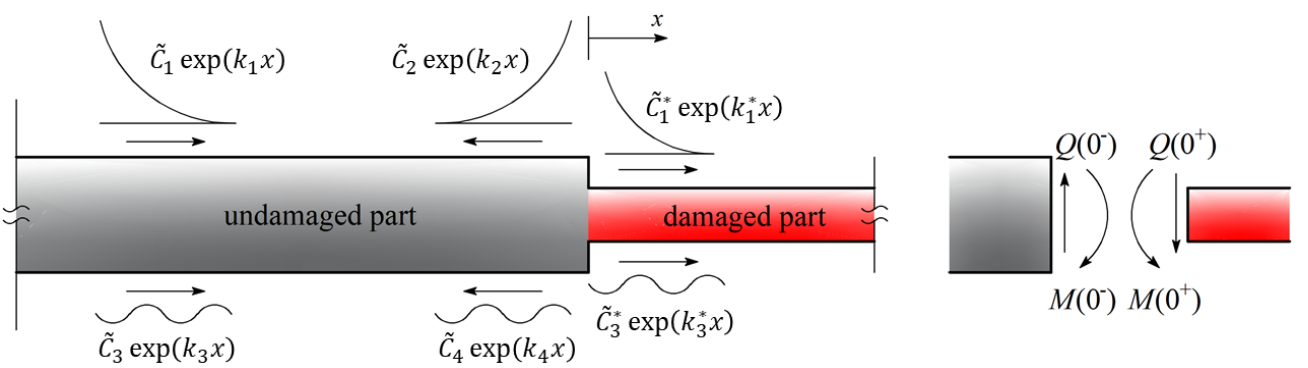

Figure 2: An infinitely long cable with a sudden change in the cross section

The response in each part $x<0$ or $x>0$ takes the form of Eq. (1) and Eq. (2) 29]. The solution at both sides of the damage interface, $x \in(-\infty, 0) \cup(0,+\infty)$, is expressed as:

$$
\begin{aligned}
& \hat{V}(x, \omega)= \begin{cases}\tilde{C}_{1} \exp \left(k_{1} x\right)+\tilde{C}_{2} \exp \left(k_{2} x\right)+\tilde{C}_{3} \exp \left(k_{3} x\right)+\tilde{C}_{4} \exp \left(k_{4} x\right) & (x<0) \\
\tilde{C}_{1}^{*} \exp \left(k_{1}^{*} x\right)+\tilde{C}_{2}^{*} \exp \left(k_{2}^{*} x\right)+\tilde{C}_{3}^{*} \exp \left(k_{3}^{*} x\right)+\tilde{C}_{4}^{*} \exp \left(k_{4}^{*} x\right) & (x>0)\end{cases} \\
& \hat{\Theta}(x, \omega)= \begin{cases}R_{1} \tilde{C}_{1} \exp \left(k_{1} x\right)+R_{2} \tilde{C}_{2} \exp \left(k_{2} x\right)+R_{3} \tilde{C}_{3} \exp \left(k_{3} x\right)+R_{4} \tilde{C}_{4} \exp \left(k_{4} x\right) & (x<0) \\
R_{1}^{*} \tilde{C}_{1}^{*} \exp \left(k_{1}^{*} x\right)+R_{2}^{*} \tilde{C}_{2}^{*} \exp \left(k_{2}^{*} x\right)+R_{3}^{*} \tilde{C}_{3}^{*} \exp \left(k_{3}^{*} x\right)+R_{4}^{*} \tilde{C}_{4}^{*} \exp \left(k_{4}^{*} x\right) & (x>0)\end{cases}
\end{aligned}
$$

where ()$^{*}$ denotes the parameter corresponding to the damaged part. Assume that an incident wave $\tilde{C}_{1} \exp \left(k_{1} x\right)+\tilde{C}_{3} \exp \left(k_{3} x\right)$ propagates from the negative side, while no excitation is present at the positive side. This implies:

$$
\tilde{C}_{2}^{*}=R_{4}^{*} \tilde{C}_{4}^{*} \equiv 0
$$


Among the wave components in Eq. 11 , $\tilde{C}_{2} \exp \left(k_{2} x\right)+\tilde{C}_{4} \exp \left(k_{4} x\right)$ and $\tilde{C}_{1}^{*} \exp \left(k_{1}^{*} x\right)+\tilde{C}_{3}^{*} \exp \left(k_{3}^{*} x\right)$ are the reflected wave and the transmitted wave, respectively (Fig. 22). The remaining 6 unknown coefficients are then determined by continuity and equilibrium [30] at the interface $x=0$. The continuity conditions are given by:

$$
\begin{aligned}
& \hat{V}\left(0^{-}, \omega\right)=\hat{V}\left(0^{+}, \omega\right) \\
& \hat{\Theta}\left(0^{-}, \omega\right)=\hat{\Theta}\left(0^{+}, \omega\right)
\end{aligned}
$$

The equilibrium conditions are given by:

$$
\begin{aligned}
M\left(0^{-}\right)-M\left(0^{+}\right) & =0 \\
Q\left(0^{-}\right)-Q\left(0^{+}\right) & =0
\end{aligned}
$$

By substituting Eq. (11) and Eq. (12) into Eq. 14- 17), a system of equations are obtained, which is written in matrix form as:

$$
\mathbf{H} \cdot \mathbf{C}=\mathbf{0}
$$

where $\mathbf{H}$ is the characteristic matrix given by:

$$
\mathbf{H}=\left[\begin{array}{cccc}
1 & R_{1} & E I R_{1} k_{1} & \kappa G A\left(k_{1}-R_{1}\right) \\
1 & R_{2} & E I R_{2} k_{2} & \kappa G A\left(k_{2}-R_{2}\right) \\
1 & R_{3} & E I R_{3} k_{3} & \kappa G A\left(k_{3}-R_{3}\right) \\
1 & R_{4} & E I R_{4} k_{4} & \kappa G A\left(k_{4}-R_{4}\right) \\
-1 & -R_{1}^{*} & -E I^{*} R_{1}^{*} k_{1}^{*} & -\kappa G A^{*}\left(k_{1}^{*}-R_{1}^{*}\right) \\
-1 & -R_{3}^{*} & -E I^{*} R_{3}^{*} k_{3}^{*} & -\kappa G A^{*}\left(k_{3}^{*}-R_{3}^{*}\right)
\end{array}\right]^{\mathrm{T}}
$$

The characteristic matrix is determined by the known conditions (i.e., material and geometric parameters, dispersion relation). The vector $\mathbf{C}$ collects the frequency-dependent wave coefficients:

$$
\mathbf{C}=\left\{\begin{array}{llllll}
\tilde{C}_{1} & \tilde{C}_{2} & \tilde{C}_{3} & \tilde{C}_{4} & \tilde{C}_{1}^{*} & \tilde{C}_{3}^{*}
\end{array}\right\}^{\mathrm{T}}
$$

The matrix $\mathbf{H}$ has the following rank:

$$
\operatorname{rank}(\mathbf{H})=4<6
$$

Eq. (18) is therefore underdetermined, and has two fundamental solutions which can be derived by elimination of variables. First, by eliminating $\tilde{C}_{1}^{*}$ and $\tilde{C}_{3}^{*}$, the reflected waves are expressed as a linear transformation of the incident waves:

$$
\left\{\begin{array}{l}
\tilde{C}_{2} \\
\tilde{C}_{4}
\end{array}\right\}=\left[\begin{array}{ll}
r_{11} & r_{12} \\
r_{21} & r_{22}
\end{array}\right]\left\{\begin{array}{l}
\tilde{C}_{1} \\
\tilde{C}_{3}
\end{array}\right\}
$$

being the first fundamental solution. $r_{11}$ and $r_{21}$ represent the coefficients of the reflected evanescent wave (or PS wave) and PB wave, respectively, for the case of an evanescent wave. $r_{12}$ and $r_{22}$ represent the coefficients of the reflected evanescent wave (or PS wave) and PB wave, respectively, for the case of an incident PB wave. Similarly, by eliminating $\tilde{C}_{2}$ and $\tilde{C}_{4}$, the transmitted waves are expressed by another linear transformation of the incident waves:

$$
\left\{\begin{array}{l}
\tilde{C}_{1}^{*} \\
\tilde{C}_{3}^{*}
\end{array}\right\}=\left[\begin{array}{ll}
t_{11} & t_{12} \\
t_{21} & t_{22}
\end{array}\right]\left\{\begin{array}{l}
\tilde{C}_{1} \\
\tilde{C}_{3}
\end{array}\right\}
$$

being the second fundamental solution. $t_{11}$ and $t_{21}$ represent the coefficients of the transmitted evanescent wave (or PS wave) and PB wave, respectively, for the case of an evanescent wave. $t_{12}$ and $t_{22}$ represent the coefficients of the transmitted evanescent wave (or PS wave) and PB wave, respectively, for the case of an incident PB wave. The closed-form solutions of these reflection and transmission coefficients are usually 
rather complicated 30. For this reason, they are calculated indirectly in this study. First, let $\tilde{C}_{1}=1$ and $\tilde{C}_{3}=0$. From Eq. 22, and Eq. 23, it follows that:

$$
\tilde{C}_{2}=r_{11}, \quad \tilde{C}_{4}=r_{21}, \quad \tilde{C}_{1}^{*}=t_{11}, \quad \tilde{C}_{3}^{*}=t_{21}
$$

By substituting Eq. (24) into Eq. (18), the reflection and transmission coefficients of the incident evanescent wave (or $\mathrm{SP}$ wave) $\tilde{C}_{1} \exp \left(k_{1} x\right)$ are derived as:

$$
\left\{\begin{array}{l}
r_{11} \\
r_{21} \\
t_{11} \\
t_{21}
\end{array}\right\}=-\mathbf{B}^{-1} \cdot\left\{\begin{array}{c}
1 \\
R_{1} \\
E I R_{1} k_{1} \\
\kappa G A\left(k_{1}-R_{1}\right)
\end{array}\right\}
$$

where the matrix $\mathbf{B}$ is:

$$
\mathbf{B}=\left[\begin{array}{cccc}
1 & 1 & -1 & -1 \\
R_{2} & R_{4} & -R_{1}^{*} & -R_{3}^{*} \\
E I R_{2} k_{2} & E I R_{4} k_{4} & -E I^{*} R_{1}^{*} k_{1}^{*} & -E I^{*} R_{3}^{*} k_{3}^{*} \\
\kappa G A\left(k_{2}-R_{2}\right) & \kappa G A\left(k_{4}-R_{4}\right) & -\kappa G A^{*}\left(k_{1}^{*}-R_{1}^{*}\right) & -\kappa G A^{*}\left(k_{3}^{*}-R_{3}^{*}\right)
\end{array}\right]
$$

Similarly, let $\tilde{C}_{1}=0$ and $\tilde{C}_{3}=1$. From Eq. 22 and Eq. 23 , it is found that:

$$
\tilde{C}_{2}=r_{12}, \quad \tilde{C}_{4}=r_{22}, \quad \tilde{C}_{1}^{*}=t_{12}, \quad \tilde{C}_{3}^{*}=t_{22}
$$

By substituting Eq. 27) into Eq. 18, the effect of the incident PB wave $\tilde{C}_{3} \exp \left(k_{3} x\right)$ is derived as:

$$
\left\{\begin{array}{c}
r_{12} \\
r_{22} \\
t_{12} \\
t_{22}
\end{array}\right\}=-\mathbf{B}^{-1} \cdot\left\{\begin{array}{c}
1 \\
R_{3} \\
E I R_{3} k_{3} \\
\kappa G A\left(k_{3}-R_{3}\right)
\end{array}\right\}
$$

From Eq. (25) and Eq. (28), the reflection and transmission coefficients at the damage interface can be calculated. A parametric study will be performed next.

\subsection{Numerical example}

In this part, a $91 \phi 7 \mathrm{~mm}$ multi-wire cable is considered. The material parameters are as follows: the elastic modulus $E=200 \mathrm{GPa}$, the density $\rho=7800 \mathrm{~kg} / \mathrm{m}^{3}$, the Poisson's ratio $\mu=0.3$. The geometric parameters are: the cross-section area $A=0.0035 \mathrm{~m}^{2}$, the moment of inertia $I_{\mathrm{z}}=1.09 \times 10^{-6} \mathrm{~m}^{4}$, the shear constant $\kappa=0.46$.

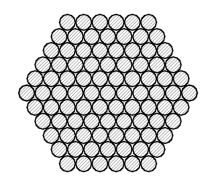

undamaged

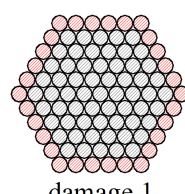

damage 1

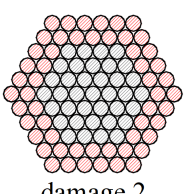

damage 2

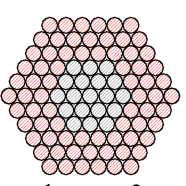

damage 3

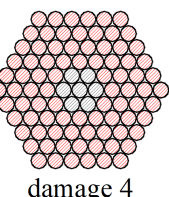

damage 4

Figure 3: Damage in the cross section of the cable

The assumed damage of the cross section, shown in Fig. 3 , represents corrosion of wires. Four different damage cases are considered and the geometric parameters of the damaged cross sections are given in Tab. 1 .

Table 1: The geometric parameters of the undamaged and damage cases

\begin{tabular}{ccccc}
\hline Case No. & Number of wires & $A\left(\mathrm{~m}^{2}\right)$ & $I\left(\mathrm{~m}^{4}\right)$ & Damage level \\
\hline Undamaged & 91 & 0.003502 & $1.09 \times 10^{-6}$ & $00.00 \%$ \\
Damage 1 & 61 & 0.002348 & $4.88 \times 10^{-7}$ & $32.97 \%$ \\
Damage 2 & 37 & 0.001424 & $1.80 \times 10^{-7}$ & $59.34 \%$ \\
Damage 3 & 19 & 0.000731 & $4.75 \times 10^{-8}$ & $79.12 \%$ \\
Damage 4 & 7 & 0.000269 & $6.48 \times 10^{-9}$ & $92.31 \%$ \\
\hline
\end{tabular}


First, the initial axial force of the cable is defined as $N_{\mathrm{x}}=500 \mathrm{MPa} \times 0.0035 \mathrm{~m}^{2}=1750 \mathrm{kN}$. The reflection and transmission coefficients are evaluated by Eq. 25) and Eq. (28), respectively (Fig. 4).
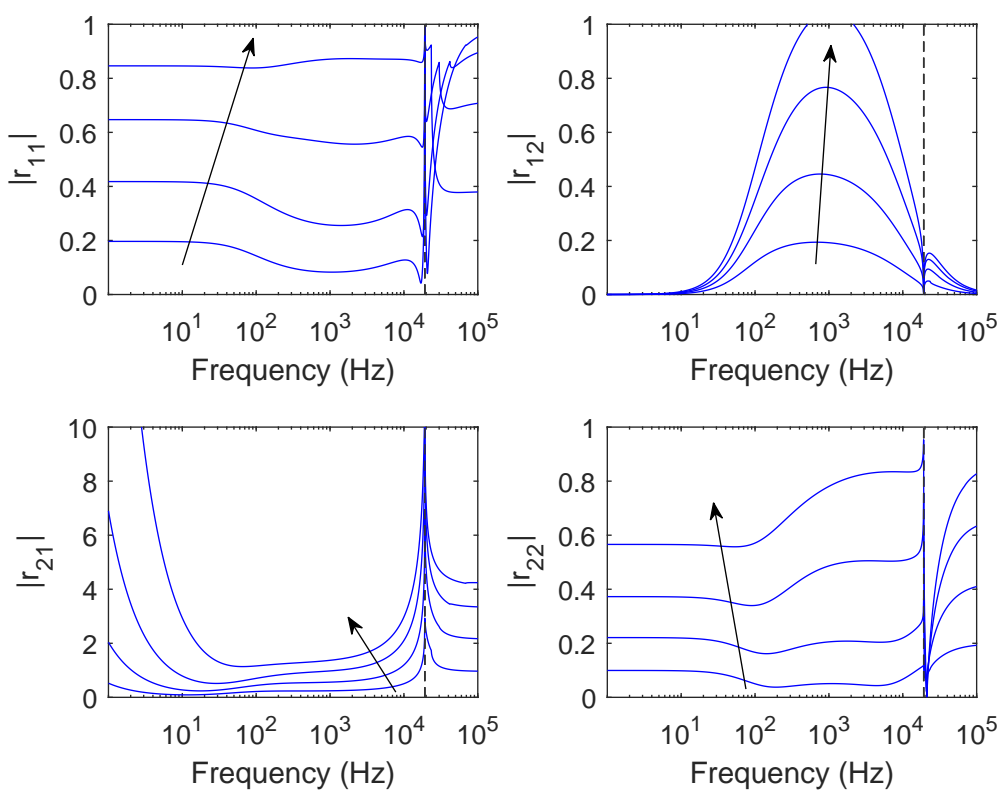

(a)
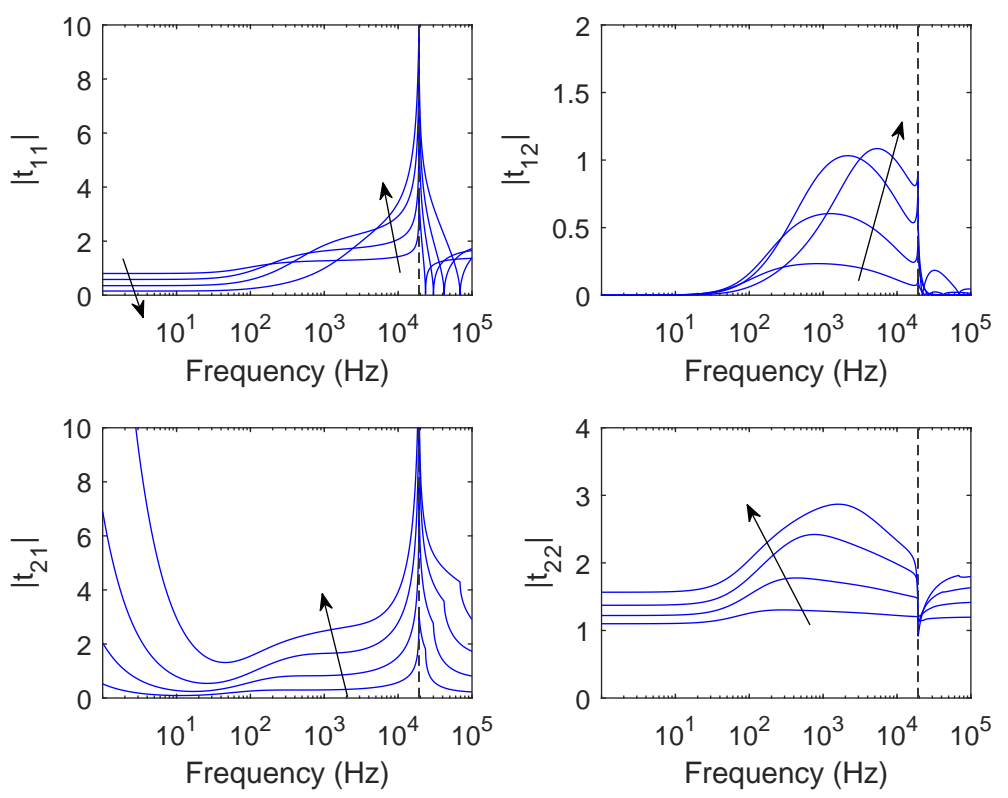

(b)

Figure 4: a) Reflection and b) transmission coefficients at the damage interface $\left(\sigma_{0}=500 \mathrm{MPa}\right)$. The arrows indicate the increase of the damage level. The vertical dashed line indicates the cut-off frequency $\omega_{\mathrm{c}}=19172 \mathrm{~Hz}$.

For the undamaged case, $t_{11}$ and $t_{22}$ have a unit value, and the other coefficients are zero. This indi- 
cates that the incident wave travels through the (undamaged) interface, with neither reflection nor mode conversion. Due to the remote location of the excitation, the incident evanescent wave $\tilde{C}_{1} \exp \left(k_{1} x\right)$ and its reflections $\tilde{C}_{2} \exp \left(k_{2} x\right)$ and $\tilde{C}_{4} \exp \left(k_{4} x\right)$ are extremely weak for the damage cases (Fig. 44 , even though the values of $r_{11}$ and $r_{21}$ are large. Note that the transmission coefficients are large (Fig. 4(b)) as well, even increasing with the damage level $\left(t_{21}\right.$ and $\left.t_{22}\right)$. This is caused by the reduced stiffness of the damaged part.

The coefficients $r_{12}$ and $r_{22}$ presented in Fig. 4(a) show the sensitivity of the reflected evanescent waves and $\mathrm{PB}$ waves to the damage interface, respectively, for the case of an incident $\mathrm{PB}$ wave. The reflected evanescent wave $\left(r_{12}\right)$ is particularly sensitive to the damage in the frequency range between 100 and 2000 $\mathrm{Hz}$ and reaches its maximum near $1000 \mathrm{~Hz}$. For the reflected PB wave $\left(r_{22}\right)$, the sensitivity is slightly lower. More importantly, the PB wave is not spatially concentrated due to its propagating behavior. It can be concluded from the above that the reflected evanescent wave has greater potential for damage identification.

With increasing axial force, the dynamic behavior of a cable becomes increasingly similar to that of a taut string (disregarding bending stiffness), especially in the lower frequency range. The intensity of the reflected evanescent wave, characterized by bending deformation, is therefore lower.

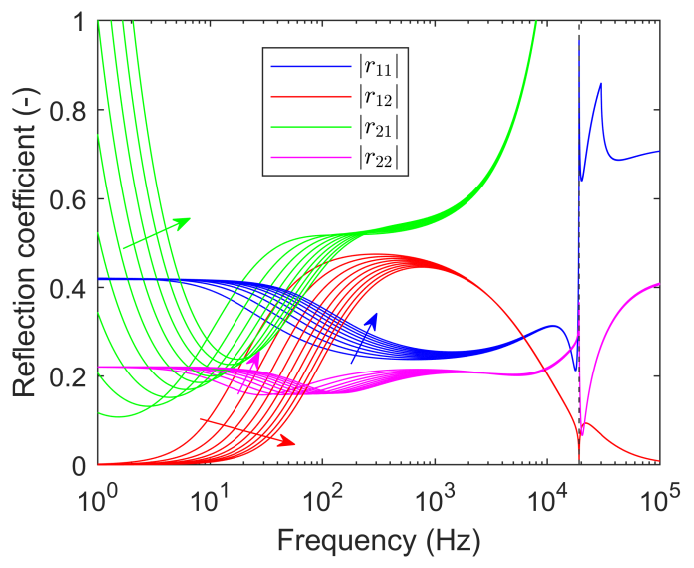

(a)

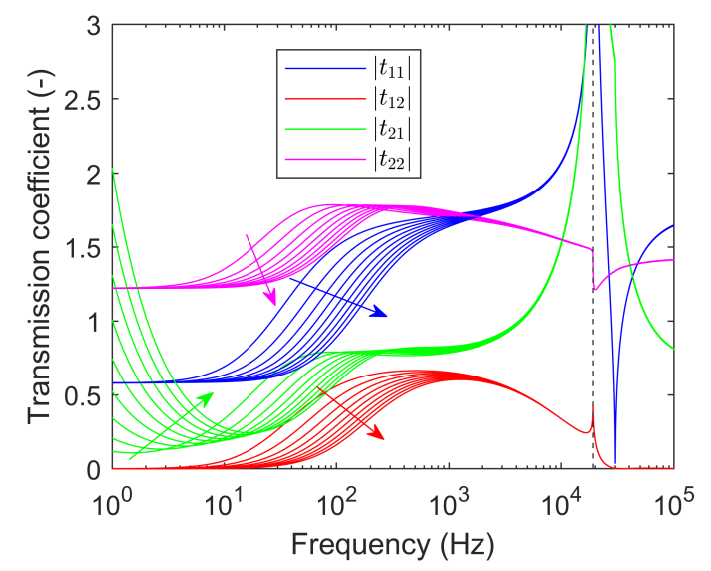

(b)

Figure 5: a) Reflections and b) transmissions at the damage interface (damage case $2, \sigma_{0}=100-500 \mathrm{MPa}$ ). The arrows indicate the increase of the cable force. The vertical dashed line indicates the cut-off frequency $\omega_{\mathrm{c}}=19172 \mathrm{~Hz}$.

In order to verify this observation, an additional parametric study is performed based on damage case 2, changing the axial stress from 100 to $500 \mathrm{MPa}$. Fig. 5 shows that the reflection of the evanescent wave $\left(r_{12}\right)$ indeed decreases with increasing axial force. It has also been proven that the high-frequency components, which characterize local bending behavior, are less sensitive to the value of axial force. Both the reflection and transmission coefficients are hardly affected by the axial force at frequencies above $300 \mathrm{~Hz}$.

The above results prove that both the intensities of reflected evanescent wave and $\mathrm{PB}$ wave are sensitive to damage. Note that the derived reflection and transmission coefficients are independent of the physical excitations and the remote boundaries of cable, as neither of them is involved in the model (Fig. 2). However, the length of cable is always finite in reality, meaning that the effects of physical boundaries should be taken into account, as well as the length of the damaged part. In the following, the spatial distribution of the wave components will be studied for this case.

\section{Damaged cable with general boundary conditions}

\subsection{Frequency-domain analytical solution}

In this part, the dynamic response of a damaged cable will be discussed for different types of boundary conditions. 


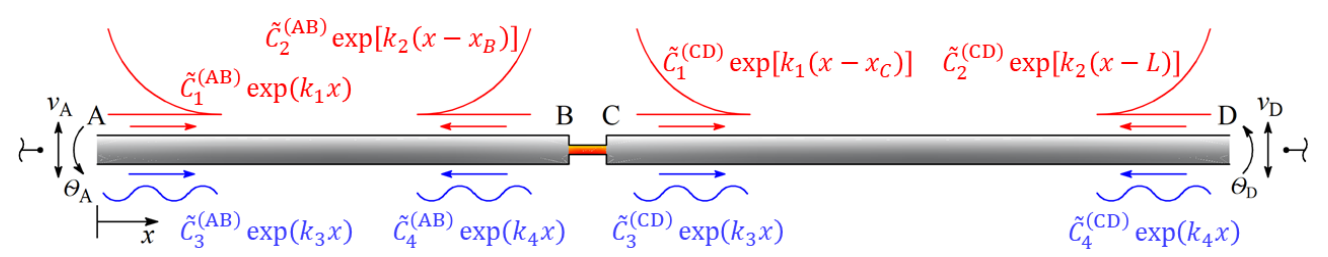

Figure 6: Model of a damaged cable with general boundary conditions

As shown in Fig. 6, a cable with finite length $L$ is considered. AB and CD are the undamaged parts, and $\mathrm{BC}$ is the damaged part. Depending on the boundary conditions considered, this model can represent either a single isolated cable, a cable connected to a main structure, or a sub-segment of a cable. Similar to Eq. (11) and Eq. (12), the frequency-domain response of this model is expressed by the following piecewise functions:

$\hat{V}(x, s)=\left\{\begin{array}{lll}\tilde{C}_{1}^{(\mathrm{AB})} \exp \left(k_{1} x\right)+\tilde{C}_{2}^{(\mathrm{AB})} \exp \left[k_{2}\left(x-x_{\mathrm{B}}\right)\right]+\tilde{C}_{3}^{(\mathrm{AB})} \exp \left(k_{3} x\right)+\tilde{C}_{4}^{(\mathrm{AB})} \exp \left(k_{4} x\right) & \left(0 \leq x<x_{\mathrm{B}}\right) \\ \tilde{C}_{1}^{(\mathrm{BC})} \exp \left[k_{1}^{*}\left(x-x_{\mathrm{B}}\right)\right]+\tilde{C}_{2}^{(\mathrm{BC})} \exp \left[k_{2}^{*}\left(x-x_{\mathrm{C}}\right)\right]+\tilde{C}_{3}^{(\mathrm{BC})} \exp \left(k_{3}^{*} x\right)+\tilde{C}_{4}^{(\mathrm{BC})} \exp \left(k_{4}^{*} x\right) & \left(x_{\mathrm{B}} \leq x<x_{\mathrm{C}}\right) \\ \tilde{C}_{1}^{(\mathrm{CD})} \exp \left(k_{1}\left(x-x_{\mathrm{C}}\right)\right)+\tilde{C}_{2}^{(\mathrm{CD})} \exp \left[k_{2}(x-L)\right]+\tilde{C}_{3}^{(\mathrm{CD})} \exp \left(k_{3} x\right)+\tilde{C}_{4}^{(\mathrm{CD})} \exp \left(k_{4} x\right) & \left(x_{\mathrm{C}} \leq x \leq x_{\mathrm{L}}\right)\end{array}\right.$

$\hat{\Theta}(x, s)=\left\{\begin{array}{lll}R_{1} \tilde{C}_{1}^{(\mathrm{AB})} \exp \left(k_{1} x\right)+R_{2} \tilde{C}_{2}^{(\mathrm{AB})} \exp \left[k_{2}\left(x-x_{\mathrm{B}}\right)\right]+R_{3} \tilde{C}_{3}^{(\mathrm{AB})} \exp \left(k_{3} x\right)+R_{4} \tilde{C}_{4}^{(\mathrm{AB})} \exp \left(k_{4} x\right) & \left(0 \leq x<x_{\mathrm{B}}\right) \\ R_{1}^{*} \tilde{C}_{1}^{(\mathrm{BC})} \exp \left[k_{1}^{*}\left(x-x_{\mathrm{B}}\right)\right]+R_{2}^{*} \tilde{C}_{2}^{(\mathrm{BC})} \exp \left[k_{2}^{*}\left(x-x_{\mathrm{C}}\right)\right]+R_{3}^{*} \tilde{C}_{3}^{(\mathrm{BC})} \exp \left(k_{3}^{*} x\right)+R_{4}^{*} \tilde{C}_{4}^{(\mathrm{BC})} \exp \left(k_{4}^{*} x\right) & \left(x_{\mathrm{B}} \leq x<x_{\mathrm{C}}\right) \\ R_{1} \tilde{C}_{1}^{(\mathrm{CD})} \exp \left(k_{1}\left(x-x_{\mathrm{C}}\right)\right)+R_{2} \tilde{C}_{2}^{(\mathrm{CD})} \exp \left[k_{2}(x-L)\right]+R_{3} \tilde{C}_{3}^{(\mathrm{CD})} \exp \left(k_{3} x\right)+R_{4} \tilde{C}_{4}^{(\mathrm{CD})} \exp \left(k_{4} x\right) & \left(x_{\mathrm{C}} \leq x \leq x_{\mathrm{L}}\right)\end{array}\right.$

where $x_{\mathrm{B}}$ and $x_{\mathrm{C}}$ are the $x$-coordinates of $\mathrm{B}$ and $\mathrm{C}$, respectively (taking $\mathrm{A}$ as the original point).

Eq. (29) and Eq. (30) contain a total of 12 unknown coefficients, requiring a system of 12 equations, established by the continuity and equilibrium conditions at the damage interface B and C, as well as the boundaries conditions at $\mathrm{A}$ and $\mathrm{D}$.

First, continuity and equilibrium at $\mathrm{B}$ and $\mathrm{C}$ provide the following 8 equations:

$$
\begin{aligned}
& \hat{V}\left(x_{\mathrm{B}}^{-}, \omega\right)=\hat{V}\left(x_{\mathrm{B}}^{+}, \omega\right) \\
& \hat{\Theta}\left(x_{\mathrm{B}}^{-}, \omega\right)=\hat{\Theta}\left(x_{\mathrm{B}}^{+}, \omega\right) \\
& M\left(x_{\mathrm{B}}, \omega\right)-M\left(x_{\mathrm{B}}^{+}, \omega\right)=0 \\
& Q\left(x_{\mathrm{B}}, \omega\right)-Q\left(x_{\mathrm{B}}^{+}, \omega\right)=0 \\
& \hat{V}\left(x_{\mathrm{C}}^{-}, \omega\right)=\hat{V}\left(x_{\mathrm{C}}^{+}, \omega\right) \\
& \hat{\Theta}\left(x_{\mathrm{C}}^{-}, \omega\right)=\hat{\Theta}\left(x_{\mathrm{C}}^{+}, \omega\right) \\
& M\left(x_{\mathrm{C}}, \omega\right)-M\left(x_{\mathrm{C}}^{+}, \omega\right)=0 \\
& Q\left(x_{\mathrm{C}}, \omega\right)-Q\left(x_{\mathrm{C}}^{+}, \omega\right)=0
\end{aligned}
$$

where the frequency-domain bending moments and shear forces are calculated as:

$$
\begin{aligned}
& Q_{\mathrm{y}}(x, \omega)=\kappa G A\left[\frac{\partial \hat{V}(x, \omega)}{\partial x}-\hat{\Theta}(x, \omega)\right] \\
& M_{\mathrm{z}}(x, \omega)=-E I \frac{\partial \hat{\Theta}(x, \omega)}{\partial x}
\end{aligned}
$$

The remaining 4 equations are obtained by considering the boundary conditions. Generally, each type of boundary condition yields two equations:

- Fixed: the displacement or rotation is constrained. (e.g. $v_{\mathrm{A}}(t)=0$ or $\theta_{\mathrm{A}}(t)=0$ )

- Free: no force or moment is present. (e.g. $Q_{\mathrm{A}}(t)=0$ or $\left.M_{\mathrm{A}}(t)=0\right)$

- Excited: the displacement or force is non-zero and known. (e.g. $v_{\mathrm{A}}(t) \neq 0$ or $\left.Q_{\mathrm{A}}(t) \neq 0\right)$

- Absorbing: the cable extends to infinity. (e.g. $\tilde{C}_{1}^{(\mathrm{AB})}=0$ or $\tilde{C}_{4}^{(\mathrm{CD})}=0$ )

In the following, two typical cases will be considered. 


\subsection{Damaged cable with fixed boundaries}

Assuming that the displacements and rotations of both boundaries are known, the 4 boundary equations take the form of:

$$
\begin{aligned}
& \hat{V}\left(x_{\mathrm{A}}, \omega\right)=\hat{V}_{\mathrm{A}} \\
& \hat{\Theta}\left(x_{\mathrm{A}}, \omega\right)=\hat{\Theta}_{\mathrm{A}} \\
& \hat{V}\left(x_{\mathrm{D}}, \omega\right)=\hat{V}_{\mathrm{D}} \\
& \hat{\Theta}\left(x_{\mathrm{D}}, \omega\right)=\hat{\Theta}_{\mathrm{D}}
\end{aligned}
$$

By substituting the analytical solution (Eq. 29-30) into the continuity and equilibrium conditions (Eq. (31 - 38), as well as the boundary conditions (Eq. (41-44), the following system of equations is established:

$$
\left[\begin{array}{ccc}
\mathbf{H}_{11} & \mathbf{0} & \mathbf{H}_{13} \\
\mathbf{H}_{21} & \mathbf{H}_{22} & \mathbf{0} \\
\mathbf{0} & \mathbf{H}_{32} & \mathbf{H}_{33}
\end{array}\right] \cdot \mathbf{C}=\mathbf{H} \cdot \mathbf{C}=\mathbf{d}
$$

where the coefficient vector $\mathbf{C}$ contains the wave coefficients, which represent the amplitude and phase of the wave components contributing to the response of the cable:

$$
\mathbf{C}=\left\{\begin{array}{llllllllllll}
\tilde{C}_{1}^{(\mathrm{AB})} & \tilde{C}_{2}^{(\mathrm{AB})} & \tilde{C}_{3}^{(\mathrm{AB})} & \tilde{C}_{4}^{(\mathrm{AB})} & \tilde{C}_{1}^{(\mathrm{BC})} & \tilde{C}_{2}^{(\mathrm{BC})} & \tilde{C}_{3}^{(\mathrm{BC})} & \tilde{C}_{4}^{(\mathrm{BC})} & \tilde{C}_{1}^{(\mathrm{CD})} & \tilde{C}_{2}^{(\mathrm{CD})} & \tilde{C}_{3}^{(\mathrm{CD})} & \tilde{C}_{4}^{(\mathrm{CD})}
\end{array}\right\}^{\mathrm{T}}
$$

the vector $\mathbf{d}$ contains the known displacements and rotations of the boundaries $\mathrm{A}$ and $\mathrm{D}$ :

$$
\mathbf{d}=\left\{\begin{array}{llllllllllll}
\hat{V}_{\mathrm{A}} & \hat{\Theta}_{\mathrm{A}} & \hat{V}_{\mathrm{D}} & \hat{\Theta}_{\mathrm{D}} & 0 & 0 & 0 & 0 & 0 & 0 & 0 & 0
\end{array}\right\}^{\mathrm{T}}
$$

In Eq. 45, $\mathbf{H}$ is the characteristic matrix, calculated by the known conditions (material and geometric parameters, dispersion relation), representing the characteristics of the system. Its sub-matrices are given by the following expressions:

$$
\begin{aligned}
& \mathbf{H}_{11}=\left[\begin{array}{cccc}
1 & \exp \left(-k_{2} x_{\mathrm{B}}\right) & 1 & 1 \\
R_{1} & R_{2} \exp \left(-k_{2} x_{\mathrm{B}}\right) & R_{3} & R_{4} \\
0 & 0 & 0 & 0 \\
0 & 0 & 0 & 0
\end{array}\right] \\
& \mathbf{H}_{13}=\left[\begin{array}{cccc}
0 & 0 & 0 & 0 \\
0 & 0 & 0 & 0 \\
\exp \left[k_{1}\left(L-x_{\mathrm{C}}\right)\right] & 1 & \exp \left(k_{3} L\right) & \exp \left(k_{4} L\right) \\
R_{1} \exp \left[k_{1}\left(L-x_{\mathrm{C}}\right)\right] & R_{2} & R_{3} \exp \left(k_{3} L\right) & R_{4} \exp \left(k_{4} L\right)
\end{array}\right] \\
& \mathbf{H}_{21}=\left[\begin{array}{cccc}
1 & 1 & 1 & 1 \\
R_{1} & R_{2} & R_{3} & R_{4} \\
E I R_{1} k_{1} & E I R_{2} k_{2} & E I R_{3} k_{3} & E I R_{4} k_{4} \\
-\kappa G A\left(k_{1}-R_{1}\right) & -\kappa G A\left(k_{2}-R_{2}\right) & -\kappa G A\left(k_{3}-R_{3}\right) & -\kappa G A\left(k_{4}-R_{4}\right)
\end{array}\right] \cdot \boldsymbol{\Lambda}_{1} \\
& \mathbf{H}_{22}=\left[\begin{array}{cccc}
-1 & -1 & -1 & -1 \\
-R_{1}^{*} & -R_{2}^{*} & -R_{3}^{*} & -R_{4}^{*} \\
-E I^{*} R_{1}^{*} k_{1}^{*} & -E I^{*} R_{2}^{*} k_{2}^{*} & -E I^{*} R_{3}^{*} k_{3}^{*} & -E I^{*} R_{4}^{*} k_{4}^{*} \\
\kappa G A^{*}\left(k_{1}^{*}-R_{1}^{*}\right) & \kappa G A^{*}\left(k_{2}^{*}-R_{2}^{*}\right) & \kappa G A^{*}\left(k_{3}^{*}-R_{3}^{*}\right) & \kappa G A^{*}\left(k_{4}^{*}-R_{4}^{*}\right)
\end{array}\right] \cdot \boldsymbol{\Lambda}_{2} \\
& \mathbf{H}_{32}=\left[\begin{array}{cccc}
1 & 1 & 1 & 1 \\
R_{1}^{*} & R_{2}^{*} & R_{3}^{*} & R_{4}^{*} \\
E I^{*} R_{1}^{*} k_{1}^{*} & E I^{*} R_{2}^{*} k_{2}^{*} & E I^{*} R_{3}^{*} k_{3}^{*} & E I^{*} R_{4}^{*} k_{4}^{*} \\
-\kappa G A^{*}\left(k_{1}^{*}-R_{1}^{*}\right) & -\kappa G A^{*}\left(k_{2}^{*}-R_{2}^{*}\right) & -\kappa G A^{*}\left(k_{3}^{*}-R_{3}^{*}\right) & -\kappa G A^{*}\left(k_{4}^{*}-R_{4}^{*}\right)
\end{array}\right] \cdot \boldsymbol{\Lambda}_{3}
\end{aligned}
$$




$$
\mathbf{H}_{33}=\left[\begin{array}{cccc}
-1 & -1 & -1 & -1 \\
-R_{1} & -R_{2} & -R_{3} & -R_{4} \\
-E I R_{1} k_{1} & -E I R_{2} k_{2} & -E I R_{3} k_{3} & -E I R_{4} k_{4} \\
\kappa G A\left(k_{1}-R_{1}\right) & \kappa G A\left(k_{2}-R_{2}\right) & \kappa G A\left(k_{3}-R_{3}\right) & \kappa G A\left(k_{4}-R_{4}\right)
\end{array}\right] \cdot \boldsymbol{\Lambda}_{4}
$$

where

$$
\begin{aligned}
& \boldsymbol{\Lambda}_{1}=\operatorname{diag}\left\{\exp \left(k_{1} x_{\mathrm{B}}\right) \quad 1 \quad \exp \left(k_{3} x_{\mathrm{B}}\right) \quad \exp \left(k_{4} x_{\mathrm{B}}\right)\right\} \\
& \boldsymbol{\Lambda}_{2}=\operatorname{diag}\left\{\begin{array}{llll}
1 & \exp \left[k_{2}^{*}\left(x_{\mathrm{B}}-x_{\mathrm{C}}\right)\right] & \exp \left(k_{3}^{*} x_{\mathrm{B}}\right) & \exp \left(k_{4}^{*} x_{\mathrm{B}}\right)
\end{array}\right\}
\end{aligned}
$$

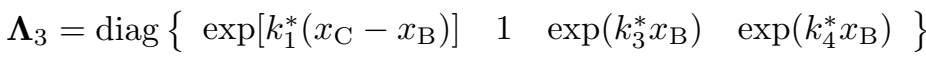

$$
\begin{aligned}
& \boldsymbol{\Lambda}_{4}=\operatorname{diag}\left\{\begin{array}{llll}
1 & \exp \left[k_{2}\left(x_{\mathrm{C}}-L\right)\right] & \exp \left(k_{3} x_{\mathrm{B}}\right) & \exp \left(k_{4} x_{\mathrm{B}}\right)
\end{array}\right\}
\end{aligned}
$$

In this part, both boundaries are assumed fixed, so that $\hat{V}_{\mathrm{A}}=\hat{\Theta}_{\mathrm{A}}=\hat{V}_{\mathrm{D}}=\hat{\Theta}_{\mathrm{D}}=0$. The coefficient vector $\mathbf{C}$ in Eq. 45 has a non-trivial solution if and only if

$$
\operatorname{det}(\mathbf{H})=0
$$

From the above characteristic equation, a series of frequencies $\omega_{j}=\{\omega \mid \operatorname{det}[\mathbf{H}(\omega)]=0\}$ are obtained, which are the natural frequencies of the cable, satisfying

$$
\operatorname{rank}\left[\mathbf{H}\left(\omega_{j}\right)\right]=11<12
$$

At each natural frequency, the fundamental solution $\mathbf{C}$ obtained from Eq. 45 represents the contributions of the wave components to the mode shape of the cable. Since the mode shapes of the cable take the form of Eq. 291, they can be decomposed as follows. The contribution of the evanescent waves to the mode shapes:

$$
\hat{V}_{\mathrm{n}}(x, \omega)= \begin{cases}\tilde{C}_{1}^{(\mathrm{AB})} \exp \left(k_{1} x\right)+\tilde{C}_{2}^{(\mathrm{AB})} \exp \left[k_{2}\left(x-x_{\mathrm{B}}\right)\right] & \left(0 \leq x<x_{\mathrm{B}}\right) \\ \tilde{C}_{1}^{(\mathrm{BC})} \exp \left[k_{1}^{*}\left(x-x_{\mathrm{B}}\right)\right]+\tilde{C}_{2}^{(\mathrm{BC})} \exp \left[k_{2}^{*}\left(x-x_{\mathrm{C}}\right)\right] & \left(x_{\mathrm{B}} \leq x<x_{\mathrm{C}}\right) \\ \tilde{C}_{1}^{(\mathrm{CD})} \exp \left(k_{1}\left(x-x_{\mathrm{C}}\right)\right)+\tilde{C}_{2}^{(\mathrm{CD})} \exp \left[k_{2}(x-L)\right] & \left(x_{\mathrm{C}} \leq x \leq x_{\mathrm{L}}\right)\end{cases}
$$

The contribution of the $\mathrm{PB}$ waves to the mode shapes:

$$
\hat{V}_{\mathrm{p}}(x, \omega)= \begin{cases}\tilde{C}_{3}^{(\mathrm{AB})} \exp \left(k_{3} x\right)+\tilde{C}_{4}^{(\mathrm{AB})} \exp \left(k_{4} x\right) & \left(0 \leq x<x_{\mathrm{B}}\right) \\ \tilde{C}_{3}^{(\mathrm{BC})} \exp \left(k_{3}^{*} x\right)+\tilde{C}_{4}^{(\mathrm{BC})} \exp \left(k_{4}^{*} x\right) & \left(x_{\mathrm{B}} \leq x<x_{\mathrm{C}}\right) \\ \tilde{C}_{3}^{(\mathrm{CD})} \exp \left(k_{3} x\right)+\tilde{C}_{4}^{(\mathrm{CD})} \exp \left(k_{4} x\right) & \left(x_{\mathrm{C}} \leq x \leq x_{\mathrm{L}}\right)\end{cases}
$$

It has been shown in Fig. 4(a) that both evanescent wave and PB wave are sensitive to damage. A numerical example will be studied next to check their sensitivity and spatial distribution.

For the cable model with fixed boundaries, an eigenvalue problem is considered in this example. The damaged cross section corresponds to damage case 1 (Tab. 1). The initial stress of the cable is $\sigma_{0}=500 \mathrm{MPa}$.

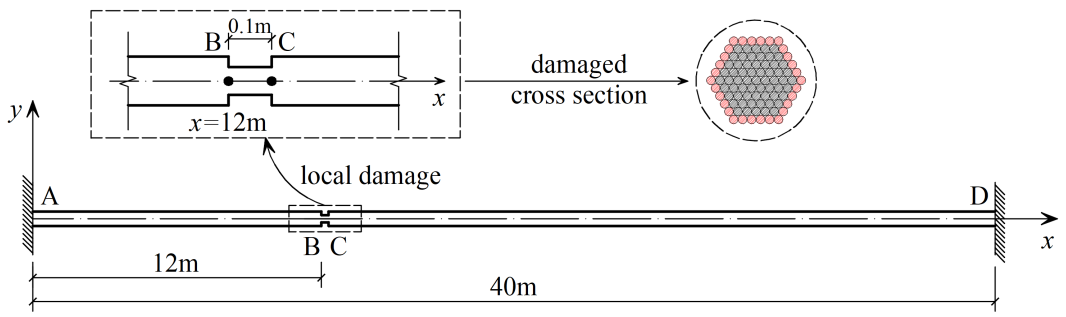

Figure 7: Model of a damaged cable with fixed boundaries

As shown in Fig. 7) the cable has a finite length $L=40 \mathrm{~m}$, and the damaged part is between $x_{\mathrm{B}}=12.0 \mathrm{~m}$ and $x_{\mathrm{C}}=12.1 \mathrm{~m}$. The boundary condition vector $\mathbf{d}=\mathbf{0}$ in Eq. (45). In order to find a series of natural frequencies $\omega_{j}$ satisfying $\operatorname{det}(\mathbf{H})=0, \operatorname{det}(\mathbf{H})$ is firstly evaluated in the frequency interval $0-1500 \mathrm{~Hz}(\mathrm{Fig}$. 8). 


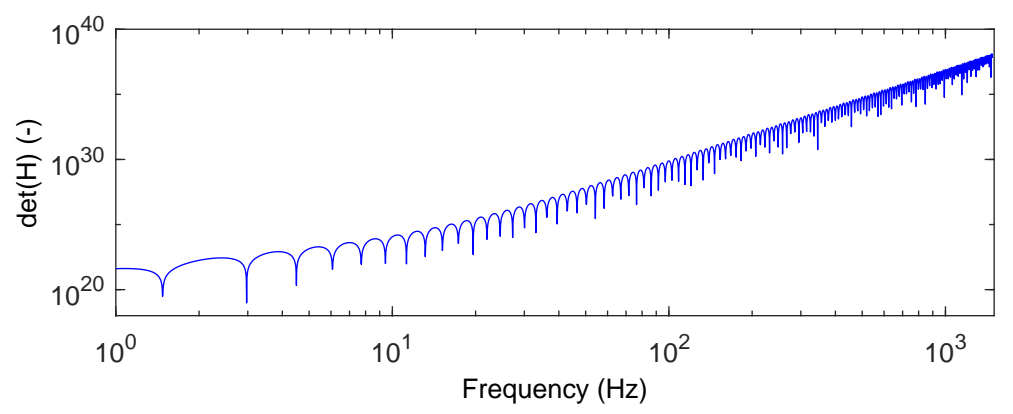

Figure 8: The determinant of the matrix $\mathbf{H}$

Each local minimum of $\operatorname{det}(\mathbf{H})$ indicates a natural frequency of the cable, and its value is found by the bisection method, solving for $\operatorname{det}(\mathbf{H}) \rightarrow 0$. The first three natural frequencies are found as $1.477 \mathrm{~Hz}, 2.972$ $\mathrm{Hz}, 4.497 \mathrm{~Hz}$. Similarly, two high-order natural frequencies near $500 \mathrm{~Hz}$ and $1000 \mathrm{~Hz}$ are calculated as 493.402 $\mathrm{Hz}$ and $1007.941 \mathrm{~Hz}$, respectively. At each of the above frequencies, a fundamental solution of the coefficient vector $\mathbf{C}$ is solved from Eq. (45), and the mode shapes (Eq. (29)) are decomposed into evanescent wave components (Eq. (60) ) and PB wave components (Eq. (61)), as shown in Fig. 9 and Fig. 10.

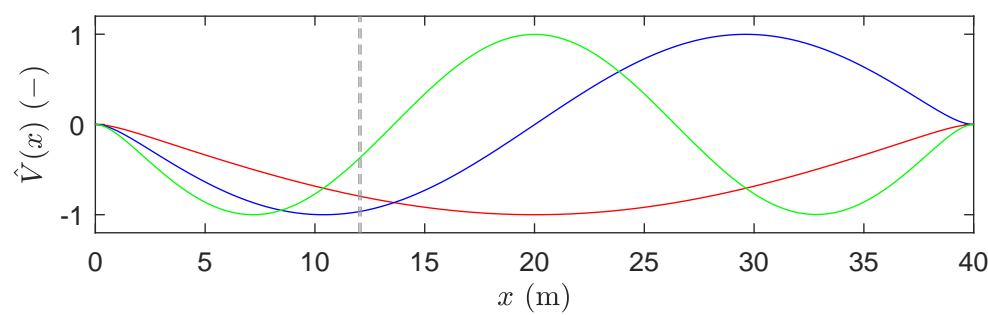

(a)

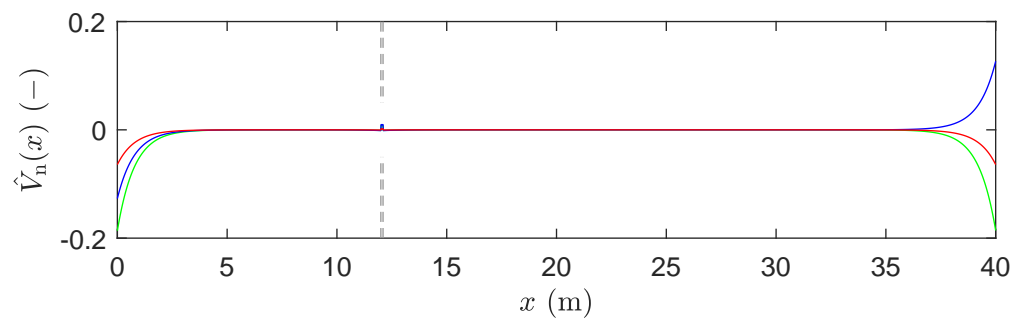

(b)

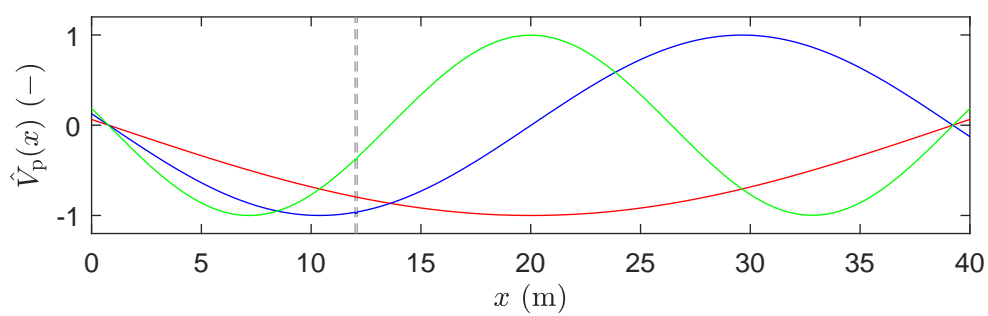

(c)

Figure 9: Lower-order mode shapes of the cable and the wave decomposition ( $-1^{\text {st }}$ order, $-2^{\text {nd }}$ order, $-3^{\text {rd }}$ order). The dashed lines indicate the damage interfaces: a) mode shapes b) evanescent waves c) PB waves 


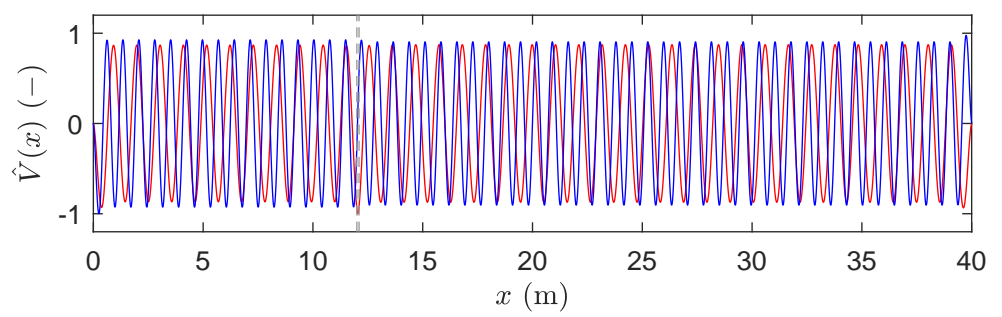

(a)

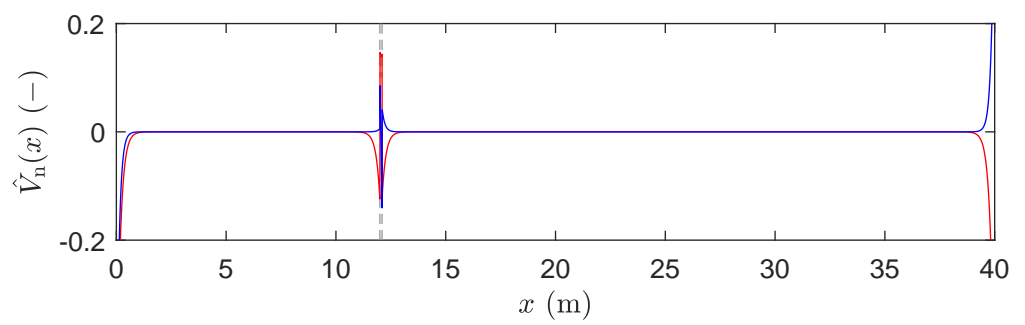

(b)

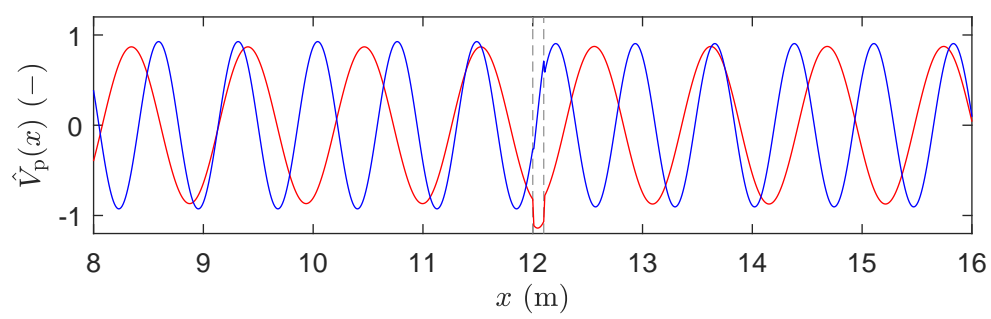

(c)

Figure 10: Higher-order mode shapes of the cable and the wave decomposition ( $-493 \mathrm{~Hz},-1008 \mathrm{~Hz})$. The dashed lines indicate the damage interfaces: a) mode shapes b) evanescent waves c) PB waves

Fig. 9(b) and Fig. 10(b) show that the evanescent wave components are spatially concentrated near the discontinuities of the cable (both the boundaries and the local damage), making each of the discontinuities easy to recognize independently. This is because the evanescent wave decays exponentially along the $x$ axis (as in Eq. (60) ). Since the evanescent wave involves a transverse motion with bending deformation, it is sensitive to the curvature at the location of discontinuity. As can be seen from Fig. 10(b), the evanescent wave component at $493 \mathrm{~Hz}$ is significantly sensitive to the damage, due to the large modal curvature (Fig. 10(c)).

The dynamic response of the cable, measured by a sensor, is the total response, which is sum of the evanescent wave and PB wave. Since the evanescent waves decays exponentially with the distance from discontinuity, it cannot be detected far away from damage location unless the resolutions of sensors are sufficiently high.

By comparing Fig. 9(a) and Fig. 9(c), as well as Fig. 10(a) and Fig. 10(c), it can be concluded that the mode shapes are dominated by $\mathrm{PB}$ waves which have a harmonic nature and propagation behavior. Therefore, it is not easy to detect damage from either mode shapes or PB wave components, despite the considerable sensitivity of PB wave shown in Fig. 4(a).

The above study is based on the fundamental solution of Eq. 45) in case of $\mathbf{d}=\mathbf{0}$. In the following, again based on Eq. 45), the wave propagation response of a damaged cable subject to a boundary excitation will be studied. 


\subsection{Wave propagation in a semi-infinite cable}

Referring again to the cable model in Fig. 6, the boundary conditions are re-defined in this part. Assume that a transverse motion is imposed at boundary A, while its rotation is constrained. The cable spatially extends to infinity along the positive $x$ axis, which is modeled by an absorbing boundary at D. For this case, Eq. 45 is still applied after being modified to satisfy the new boundary conditions.

The transverse motion at the boundary $\mathrm{A}$ is known, so that $\hat{V}_{\mathrm{A}} \neq 0$ and $\hat{\Theta}_{\mathrm{A}}=0$ in the vector $\mathbf{d}$ (Eq. (47)). For the absorbing boundary D, the coefficients $\tilde{C}_{2}^{(\mathrm{CD})}$ and $\tilde{C}_{4}^{(\mathrm{CD})}$, contained in the vector $\mathbf{C}$, are zero. Eq. (43) and Eq. (44) are eliminated. In order to satisfy the above conditions, Eq. (45) is modified as:

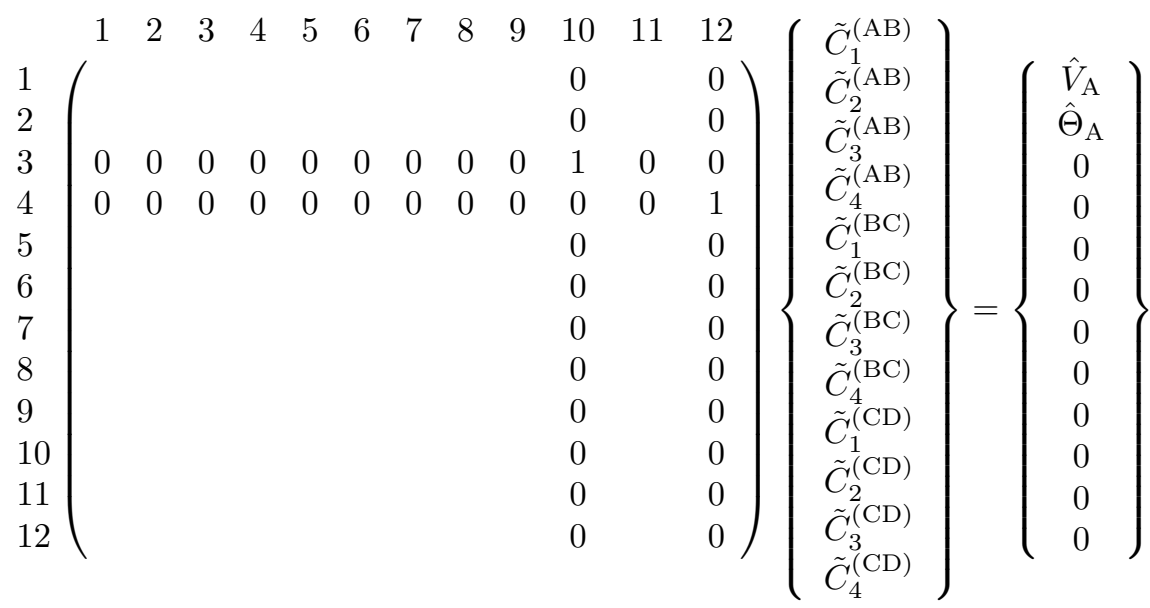

where the other elements of the matrix at the left hand side are the same as those included in the previous $\mathbf{H}$ (Eq. (45)). Eq. (62) presents a fully determined property, and a unique solution of $\mathbf{C}$ is obtained at each frequency. The frequency-domain response of the cable, taking the form of Eq. (29), will now be decomposed into evanescent wave components (Eq. 60) ) and PB wave components (Eq. (61)).

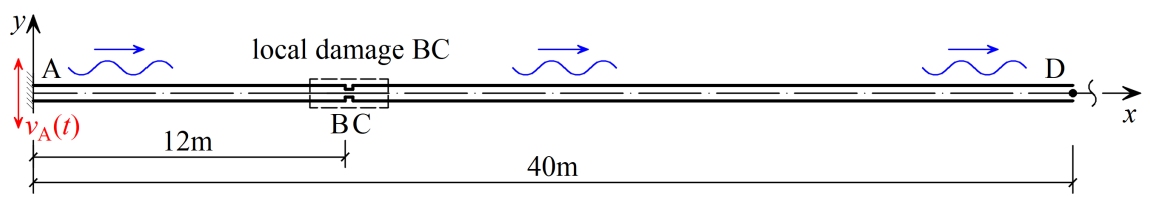

Figure 11: Model of a damaged cable excited by a boundary displacement

The cable model in Section 4.2 is again considered as an example. As shown in Fig. 11, the transverse motion $v_{\mathrm{A}}(t)$ is imposed at the left boundary A. An absorbing boundary is assumed at $\mathrm{D}$, indicating that the cable spatially extends to infinity along the positive $x$ axis. The boundary excitation is assumed to be a Dirac Delta function:

$$
v_{\mathrm{A}}(t)=\delta(t)
$$

which represents a general case. In the frequency domain, the Fourier transform of Eq. (63):

$$
\hat{V}_{\mathrm{A}}(\omega)=1
$$

represents a unit displacement at each frequency. The coefficient vector $\mathbf{C}$ is then calculated from Eq. 62. By substituting C into Eq. (29), Eq. (60), Eq. (61), the frequency-domain response of the cable, and the wave components are calculated, shown in Fig. 12. 


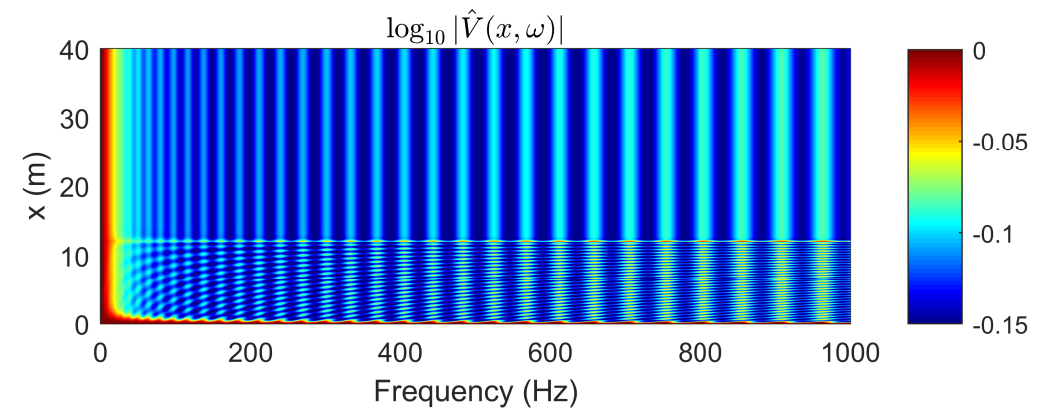

(a)

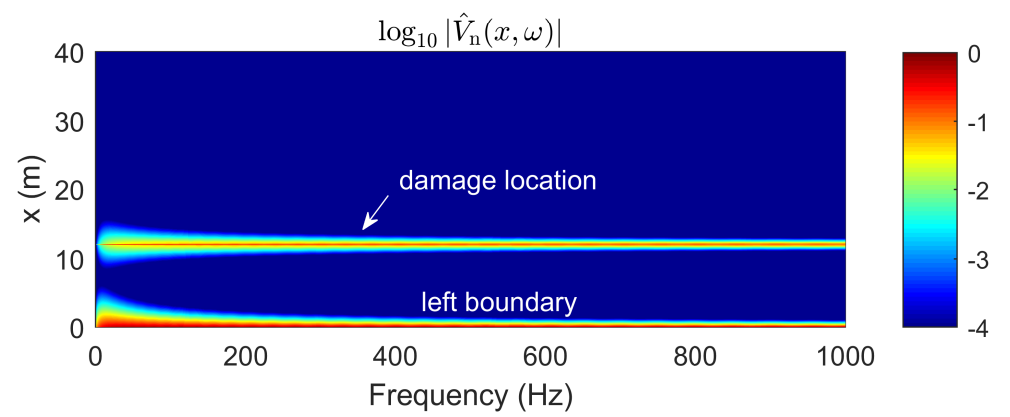

(b)

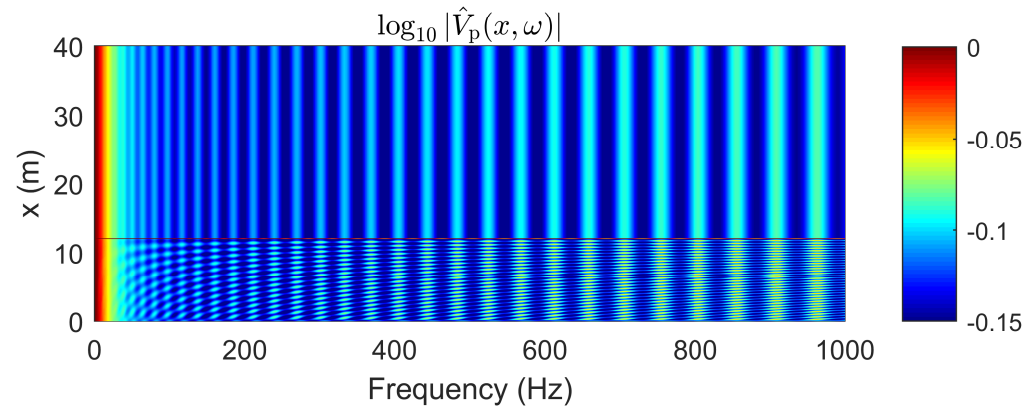

(c)

Figure 12: Frequency response of the cable imposed by the transverse motion of a Dirac Delta function: a) transverse displacement field b) evanescent wave component c) PB wave component

From Fig. 12(b), it is again found that the contributions from the evanescent waves are concentrated near discontinuities. In order to quantify the effect of the local damage, the results in Fig. 12 are studied in more detail. First, the wave components at the damage location $x=12 \mathrm{~m}$ and the undamaged location $x=30 \mathrm{~m}$ are compared in Fig. 13 


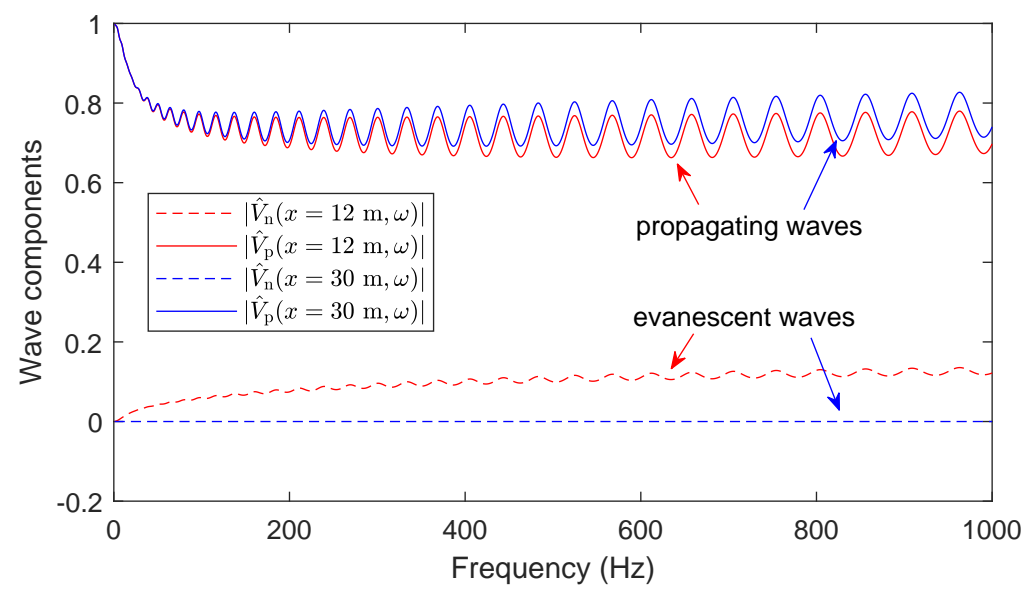

Figure 13: Wave components at the damaged $(x=12 \mathrm{~m})$ and undamaged $(x=30 \mathrm{~m})$ locations

Fig. 13 shows that the evanescent wave is significant at the damage location, getting even more intensive with increasing frequency. At the location $x=12 \mathrm{~m}$, the ratio between the evanescent wave and the PB wave is around 0.2 , which is consistent with the reflection coefficient $\left|r_{12}\right|$ in Fig. 4(a). The PB waves, which characterize the global response of the cable, on the other hand, are less sensitive to the local damage.

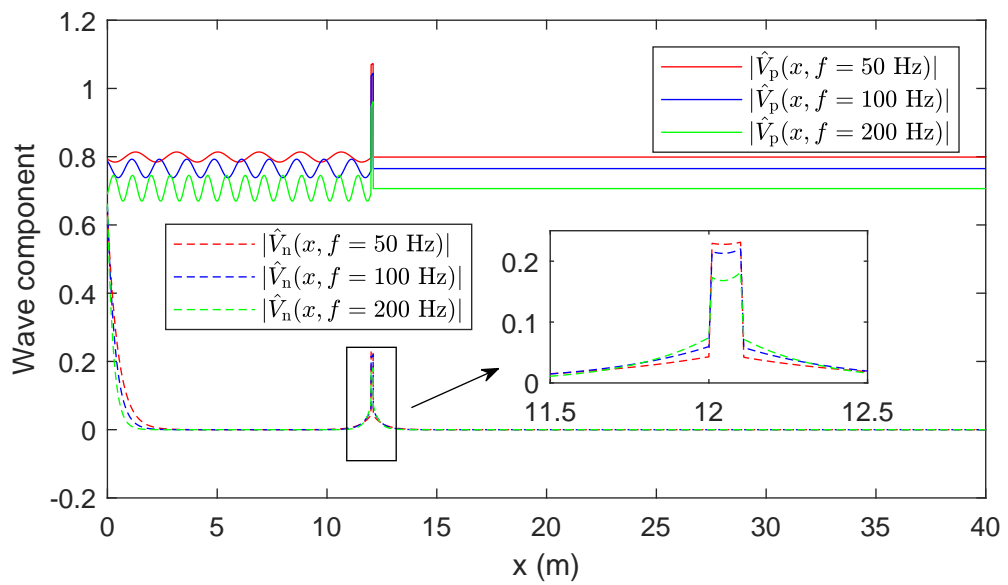

Figure 14: The spatial distributions of the wave components at $50 \mathrm{~Hz}, 100 \mathrm{~Hz}$ and $200 \mathrm{~Hz}$ (The solid lines and the dashed lines represent the evanescent waves and the $\mathrm{PB}$ waves, respectively)

Next, the spatial distribution of the wave components at the frequencies $50 \mathrm{~Hz}, 100 \mathrm{~Hz}, 200 \mathrm{~Hz}$ are compared in Fig. 14. The spatial distributions of the evanescent waves near the discontinuities, i.e., $x=0 \mathrm{~m}$ (left boundary), $x=12 \mathrm{~m}$ and $x=12.1 \mathrm{~m}$ (damage interfaces), are of particular interest. At higher frequencies, the reflected evanescent waves (at the outer side of the damage) are more intensive, which is consistent with Fig. 13. The spatial decay of higher-frequency evanescent wave is faster, however, due to 8 the larger value of wavenumber.

In order to identify the area where the evanescent wave can potentially be detected, Fig. 15 shows the locations where the evanescent wave generated by the damage satisfies the following condition which is here assumed sufficient for detection:

$$
x \in\left\{x|| \hat{V}_{\mathrm{n}}(x, \omega) \mid>0.01\right\}
$$




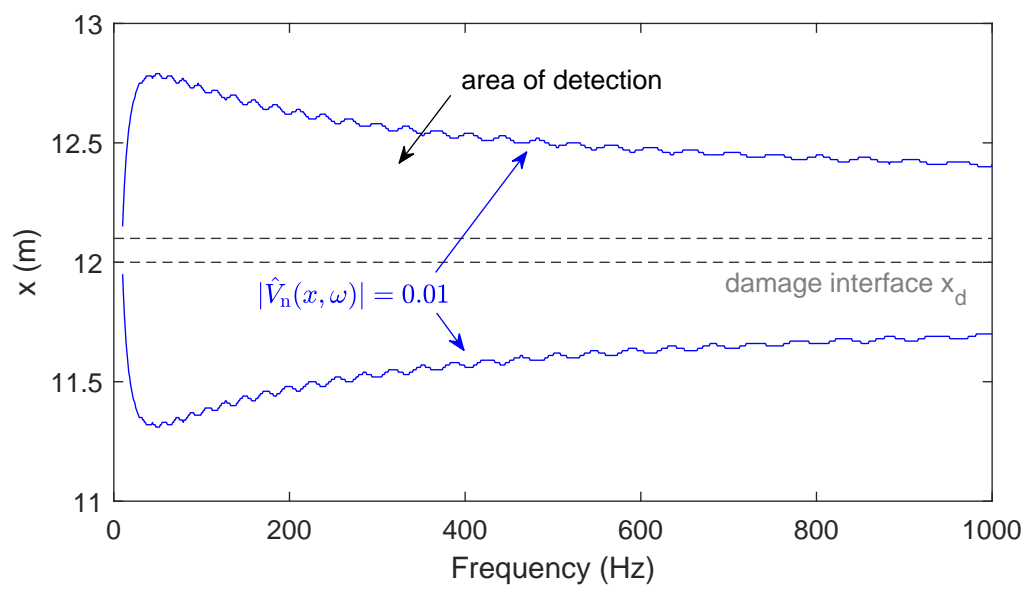

Figure 15: The suitable area for the detection of evanescent wave

In the frequency range between 50 and $100 \mathrm{~Hz}$, the detection area reduces with increasing frequency. The sensitivity of the lower-frequency evanescent wave to local damage is lower than the higher frequency components, however (Fig. 14p. It can be concluded that a trade-off is needed between a large detection area and a high intensity of the detected evanescent wave.

From the above study, it is concluded that local damage can in principle be identified from evanescent wave components. In practical cases, however, the displacement field shown in Fig. 12(a) is difficult to measure. Instead, the total response, which consists of evanescent wave and PB wave components, can only be measured at a few observation points. A suitable methodology for signal processing is therefore needed to decompose such measured response into the proposed wave components. This will be studied next.

\section{Damage identification based on wave decomposition}

\subsection{Estimation of wave coefficients}

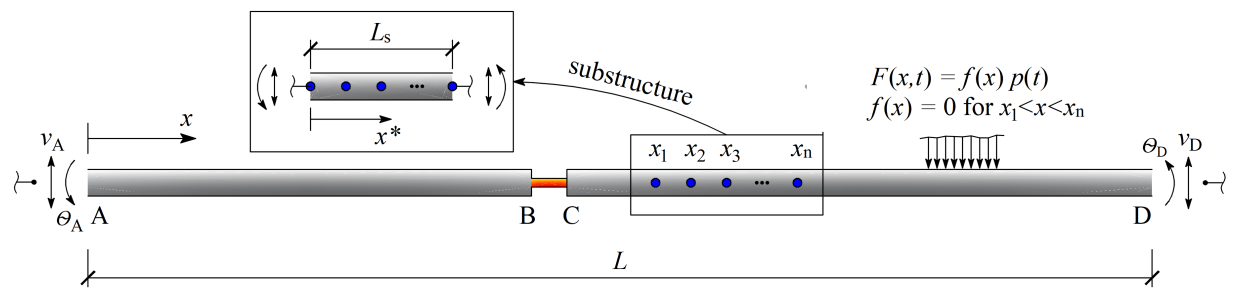

Figure 16: Model of a damaged cable excited by boundary motions and external force

As shown in Fig. 16, suppose that a few sensors are located at:

$$
\mathbf{x}=\left\{\begin{array}{llll}
x_{1} & x_{2} & \cdots & x_{n}
\end{array}\right\}^{\mathrm{T}}
$$

First of all, the substructure between $x=x_{1}$ and $x=x_{\mathrm{n}}$ is defined, with length $L_{\mathrm{s}}=\left|x_{\mathrm{n}}-x_{1}\right|$. As long as neither local damage nor external forces are contained in $\left(x_{1}, x_{\mathrm{n}}\right)$, the substructure is an undamaged cable, excited by boundary displacements (as well as rotations), regardless of the actual physical excitation of the cable, i.e., the imposed motion at the boundaries $\mathrm{A}$ and $\mathrm{D}$, the external force $F(x, t)$. The transverse motion of the substructure therefore takes the form of Eq. (1) in the frequency domain. The four wave coefficients $\tilde{C}_{1}, \tilde{C}_{2}, \tilde{C}_{3}, \tilde{C}_{4}$ are unknown, requiring 4 equations, which are obtained by the consideration 
of the measurements. By applying the Laplace transform, the measured response is transformed into the frequency domain:

$$
\hat{\mathbf{V}}_{\mathrm{m}}=\left\{\hat{V}_{\mathrm{m}}\left(x_{1}^{*}\right), \hat{V}_{\mathrm{m}}\left(x_{2}^{*}\right), \cdots, \hat{V}_{\mathrm{m}}\left(x_{n}^{*}\right)\right\}^{\mathrm{T}}
$$

where $x_{j}^{*}=x_{j}-x_{1}(j=1,2, \cdots, n)$ are the locations of the measurement points with the local coordinate system defined at the level of the substructure. In order to avoid numerical problems arising from the singularity of the characteristic matrix, the wave coefficients $\tilde{C}_{2}$ and $\tilde{C}_{4}$ in Eq. 11$)$ are replaced by $\tilde{C}_{2} \exp \left(-k_{2} L_{\mathrm{s}}\right)$ and $\tilde{C}_{4} \exp \left(-k_{4} L_{\mathrm{s}}\right)$, respectively. The frequency-domain analytical solution of the substructures is then re-written as:

$$
\hat{V}\left(x^{*}, s\right)=\tilde{C}_{1} \exp \left(k_{1} x^{*}\right)+\tilde{C}_{2} \exp \left[k_{2}\left(x^{*}-L_{\mathrm{s}}\right)\right]+\tilde{C}_{3} \exp \left(k_{3} x^{*}\right)+\tilde{C}_{4} \exp \left[k_{4}\left(x^{*}-L_{\mathrm{s}}\right)\right]
$$

Assuming that the measured response (Eq. (67)) equals the analytical solution (Eq. (68), a system of equations is established as:

$$
\mathbf{H} \cdot \tilde{\mathbf{C}}^{(\mathrm{es})}=\hat{\mathbf{V}}_{\mathrm{m}}
$$

where the coefficient vector, containing the estimated wave coefficients of Eq. [68 is given as:

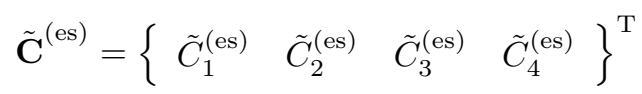

The characteristic matrix, determined by the known conditions, is given as:

$$
\mathbf{H}=\left[\begin{array}{cccc}
\exp \left(k_{1} x_{1}^{*}\right) & \exp \left[k_{2}\left(x_{1}^{*}-L_{s}\right)\right] & \exp \left(k_{3} x_{1}^{*}\right) & \exp \left[k_{4}\left(x_{1}^{*}-L_{s}\right)\right] \\
\exp \left(k_{1} x_{2}^{*}\right) & \exp \left[k_{2}\left(x_{2}^{*}-L_{s}\right)\right] & \exp \left(k_{3} x_{2}^{*}\right) & \exp \left[k_{4}\left(x_{2}^{*}-L_{s}\right)\right] \\
\vdots & \vdots & \vdots & \vdots \\
\exp \left(k_{1} x_{n}^{*}\right) & \exp \left[k_{2}\left(x_{n}^{*}-L_{s}\right)\right] & \exp \left(k_{3} x_{n}^{*}\right) & \exp \left[k_{4}\left(x_{n}^{*}-L_{s}\right)\right]
\end{array}\right]
$$

The wave coefficients are obtained by matrix inversion or the Least Squares Method:

$$
\tilde{\mathbf{C}}^{(\mathrm{es})}=\mathbf{H}^{-1} \hat{\mathbf{V}}_{\mathrm{m}}(n=4) \quad \text { or } \quad \tilde{\mathbf{C}}^{(\mathrm{es})}=\left(\mathbf{H}^{\mathrm{T}} \mathbf{H}\right)^{-1} \mathbf{H}^{\mathrm{T}} \hat{\mathbf{V}}_{\mathrm{m}}(n>4)
$$

It is interesting to note that Eq. $\sqrt{72}$ in fact represents a linear transform of the measured response $\hat{\mathbf{V}}_{\mathrm{m}}$, from the frequency domain to the wavenumber domain.

By substituting $\tilde{\mathbf{C}}^{(\text {es) }}$ back into Eq. 68 , the transverse motion of the substructure is reconstructed in the frequency domain, expressed in the local coordinate system as:

$$
\hat{V}\left(x^{*}, s\right)=\tilde{C}_{1}^{(\mathrm{es})} \exp \left(k_{1} x^{*}\right)+\tilde{C}_{2}^{(\mathrm{es})} \exp \left[k_{2}\left(x^{*}-L_{\mathrm{s}}\right)\right]+\tilde{C}_{3}^{(\mathrm{es})} \exp \left(k_{3} x^{*}\right)+\tilde{C}_{4}^{(\mathrm{es})} \exp \left[k_{4}\left(x^{*}-L_{\mathrm{s}}\right)\right]
$$

where $\tilde{C}_{1}^{(\mathrm{es})}$ and $\tilde{C}_{2}^{(\mathrm{es})}$ represent the evanescent waves at $x^{*}=0$ (decaying along the positive direction) and $x^{*}=L_{\mathrm{s}}$ (decaying along the negative direction), respectively. $\tilde{C}_{3}^{(\mathrm{es})}$ and $\tilde{C}_{4}^{(\mathrm{es})}$ represent the PB waves at $x^{*}=0$ (propagating along the positive direction) and $x^{*}=L_{\mathrm{s}}$ (propagating along the negative direction), respectively (Fig. 17).

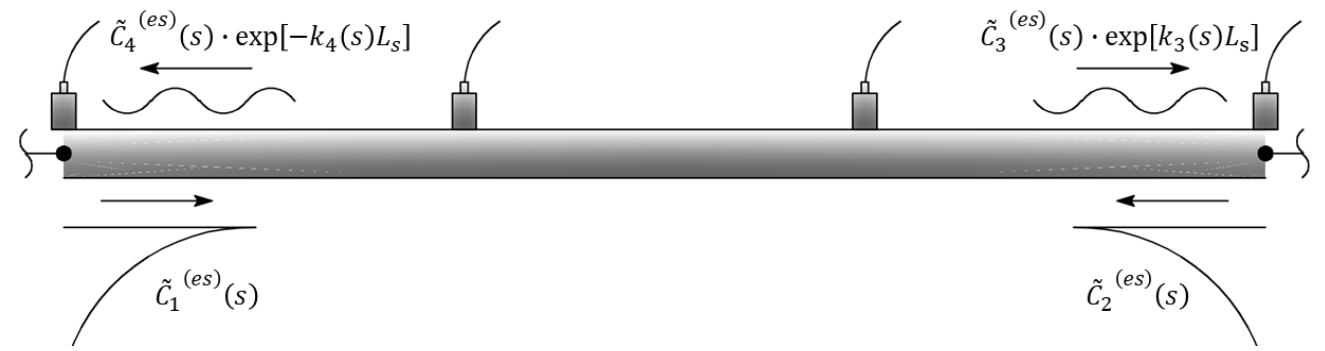

Figure 17: Reflections of the evanescent waves at the boundaries of the substructure 
From $\tilde{C}_{1}^{(\mathrm{es})}$ and $\tilde{C}_{2}^{(\mathrm{es})}$, the discontinuities at the left and right side to the substructure, respectively, can be identified. The absolute values of $\tilde{C}_{1}^{(\text {es) }}$ and $\tilde{C}_{2}^{(\text {es })}$ are affected by the frequency response of cable, and cannot be used for damage quantification. In order to derive a damage indicator depending only on the characteristics of the local discontinuity, the reflection coefficients of evanescent wave (REW) for incident $\mathrm{PB}$ waves are estimated from the reconstructed response of the substructure (Fig. 17):

$$
\begin{aligned}
{\left[r_{12}^{(\mathrm{es})}\right]_{\mathrm{L}}(s) } & =\frac{\tilde{C}_{1}^{(\mathrm{es})}(s)}{\tilde{C}_{4}^{(\mathrm{es})}(s) \exp \left[-k_{4}(s) L_{s}\right]} \\
{\left[r_{12}^{(\mathrm{es})}\right]_{\mathrm{R}}(s) } & =\frac{\tilde{C}_{2}^{(\mathrm{es})}(s)}{\tilde{C}_{3}^{(\mathrm{es})}(s) \exp \left[k_{3}(s) L_{s}\right]}
\end{aligned}
$$

where $\left[r_{12}^{(\text {es })}\right]_{\mathrm{L}}$ indicates the REW at $x^{*}=0$ for the incident PB wave along the negative direction, $\left[r_{12}^{(\mathrm{es})}\right]_{\mathrm{R}}$ indicates the REW at $x^{*}=L_{\mathrm{s}}$ for the incident $\mathrm{PB}$ wave along the positive direction. The estimated $\left[r_{12}^{(\mathrm{es})}\right]_{\mathrm{L}}$ and $\left[r_{12}^{(\mathrm{es})}\right]_{\mathrm{R}}$, which correspond to the coefficient $r_{12}$ of Eq. 22 , depend only on the characteristics of the discontinuity near the substructure, i.e., they depend on neither physical excitation nor the global response of cable. This renders them suitable for local damage identification.

\subsection{Numerical example}

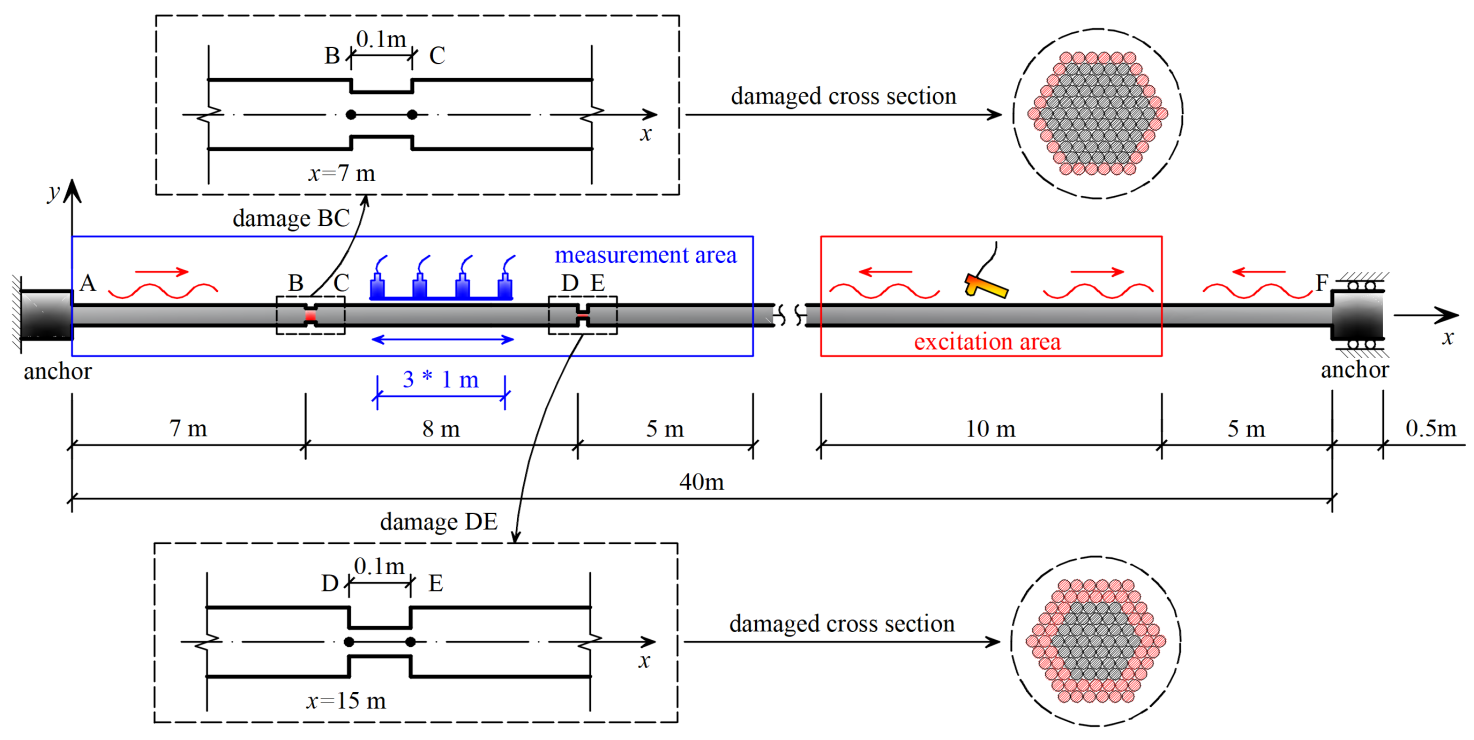

Figure 18: The boundary excitation and the measurement

In this part, the cable model of Section 4.2 is again considered. Particularly for this example, two anchors are modeled at both end points of the cable, as shown in Fig. 18. The length of each anchor is $L_{\mathrm{a}}=0.5 \mathrm{~m}$. The cross section of the anchors is assumed to be a circle with diameter $D_{\mathrm{a}}=0.1 \mathrm{~m}$, so the cross-section area is $A_{\mathrm{a}}=0.0078 \mathrm{~m}^{2}$ and the moment of inertia is $I_{\mathrm{a}}=4.909 \times 10^{-6} \mathrm{~m}^{4}$. Since the evanescent waves are spatially concentrated, multiple local damages at different locations can be detected independently, as long as the areas of detection do not overlap. At the locations $x=7 \mathrm{~m}$ and $x=15 \mathrm{~m}$, local damages $\mathrm{BC}$ and DE are considered, with the damage levels of case 1 and case 2 in Tab. 1. respectively. At the right boundary F, a constant tensile force $N_{\mathrm{x}}=1750 \mathrm{kN}$ is applied.

Tab.2 and Fig. 19 compare the natural frequencies and mode shapes of the cable before and after damage, respectively, taking into account the static configuration under the own weight loading, as calculated by means of the finite element method. 
Table 2: The natural frequencies of the cable model $(\mathrm{Hz})$

\begin{tabular}{cccccc}
\hline Order & 1 & 2 & 3 & 5 & 10 \\
\hline Undamaged & 3.190 & 6.386 & 9.597 & 16.090 & 33.055 \\
Damaged & 3.193 & 6.395 & 9.611 & 16.094 & 33.082 \\
Relative change & $0.09 \%$ & $0.14 \%$ & $0.15 \%$ & $0.02 \%$ & $0.08 \%$ \\
\hline
\end{tabular}

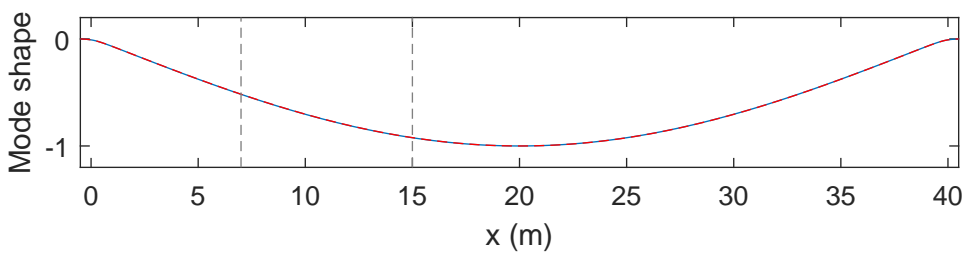

(a)

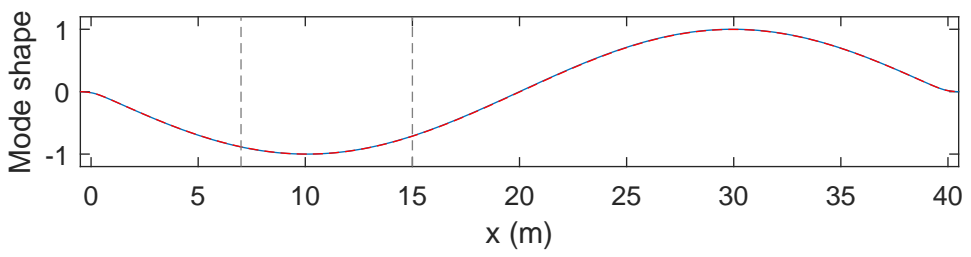

(b)

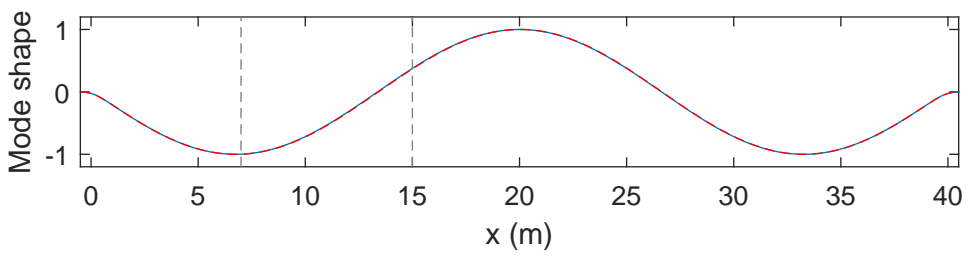

(c)

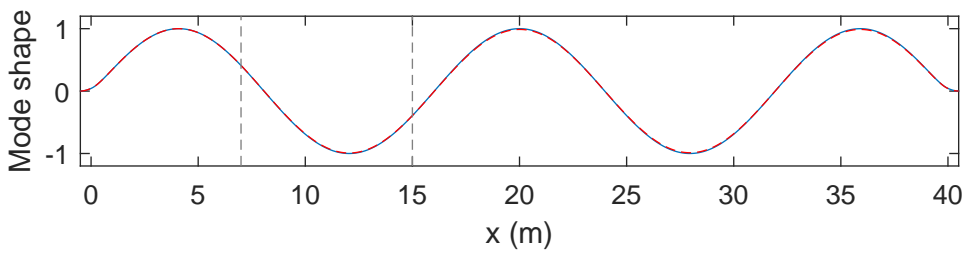

(d)

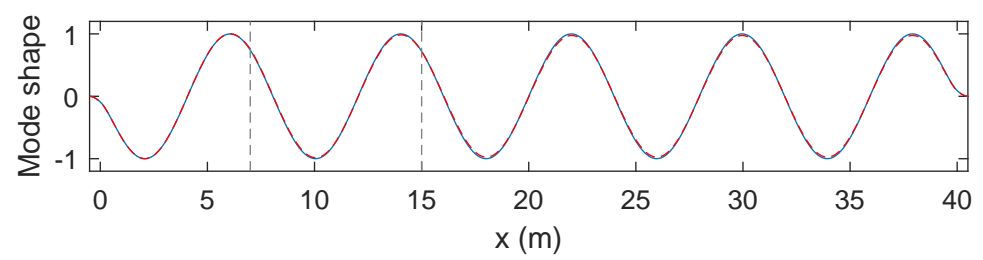

(e)

Figure 19: The mode shapes of the cable model ( - undamaged - - - damaged). The gray dashed lines indicate the damage locations: a) $1^{\text {st }}$ mode b) $2^{\text {nd }}$ mode c) $3^{\text {rd }}$ mode d) $5^{\text {th }}$ mode e) $10^{\text {th }}$ mode 
Interestingly, the transverse stiffness of the cable, which is mainly provided by the cable force, hardly changes after damage. As a consequence, the natural frequencies of the cable model actually slightly increase due to the loss of mass in the damaged parts. Therefore, local damage cannot be identified from changes in the natural frequencies in such a case. Additionally, the mode shapes before and after damage are compared in Fig. 19, showing that the local damages have little effect to the mode shapes.

In this simulated experiment, the reflection coefficients $\left[r_{12}^{(\mathrm{es})}\right]_{\mathrm{L}}$ and $\left[r_{12}^{(\mathrm{es})}\right]_{\mathrm{R}}$ will be estimated by means of the proposed methodology, and will be applied for local damage identification. A sensor array, composed of 4 accelerometers with a spacing of $\Delta x=1 \mathrm{~m}$, is applied. Assuming that the sensor array can be moved along the cable, the experimental procedure is as follows:

1. The sensor array is initially located at $\mathbf{x}=\{0 \mathrm{~m}, 1 \mathrm{~m}, 2 \mathrm{~m}, 3 \mathrm{~m}\}^{\mathrm{T}}$;

2. An unmeasured hammer impact is applied at an arbitrary location in a certain area;

3. The acceleration response is measured by the sensor array;

4. The sensor array is moved in the $x$ positive direction by $0.1 \mathrm{~m}$;

5. Repeat step 2 to 4 , until the sensor array reaches $\mathbf{x}=\{17 \mathrm{~m}, 18 \mathrm{~m}, 19 \mathrm{~m}, 20 \mathrm{~m}\}^{\mathrm{T}}$.

It has been shown that an evanescent wave is generated at each discontinuity. Therefore, the discontinuity of the shear force produced by the hammer impact generates evanescent wave as well, which may interfere with the detection of the evanescent waves generated from damage, in particular when the location of impact is close to the sensors. Due to the exponential decay of the evanescent wave generated by the hammer impact, it will not interfere with the damage identification as long as the location of the impact is far away from the sensors. For this reason, each hammer impact applied to the cable model is modeled as:

$$
p(x, t)=A_{\mathrm{h}} \cdot h(t) \cdot \delta\left(x-x_{\mathrm{h}}\right)
$$

where $A_{\mathrm{h}}$ is the amplitude of the impact force, which is an arbitrary value between 300 and $500 \mathrm{~N}$ in this example. $x_{\mathrm{h}}$ is the location of the hammer impact, which is an arbitrary value between 25 and $35 \mathrm{~m}$ in this example. $h(t)$ is a linear impact signal with a unit amplitude starting from $t_{\mathrm{s}}=0.5 \mathrm{~s}$, and the total duration is $t_{\mathrm{d}}=4 \mathrm{~ms}$.

In this experiment, the sampling frequency $f_{\mathrm{s}}=1000 \mathrm{~Hz}$, and the total number of samples $N_{\mathrm{s}}=2^{11}=$ 2048. The time-domain signal and the linear spectrum of $h(t)$ are shown in Fig. 20

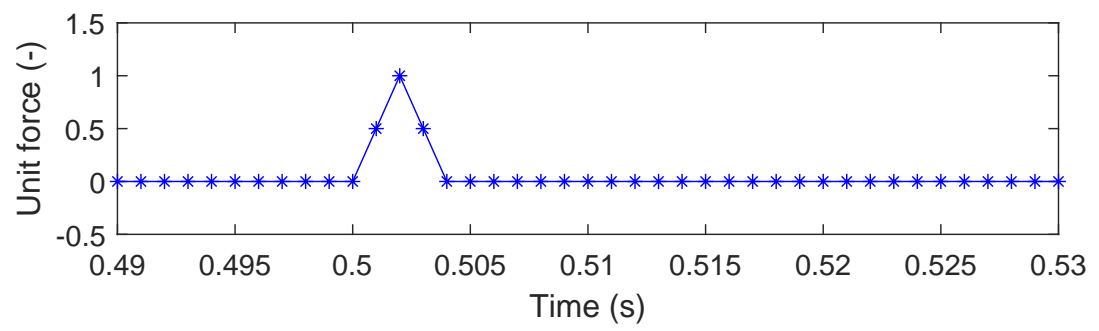

(a)

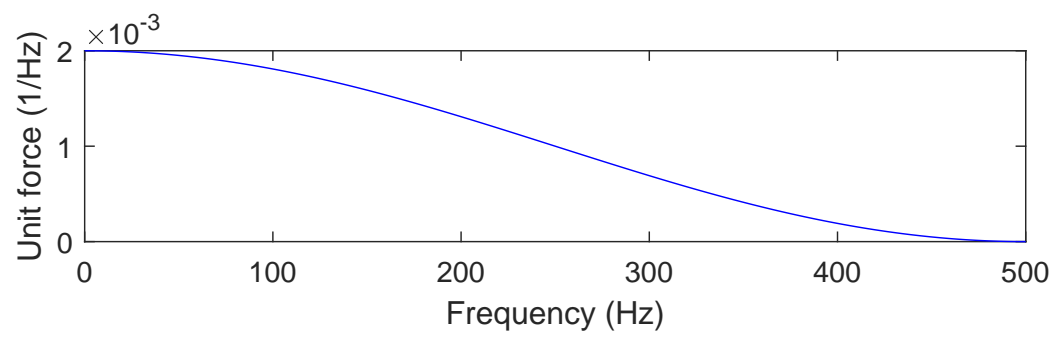

(b)

Figure 20: Unit impact $h(t)$ : a) time-domain signal b) linear spectrum 
The dynamic response of the cable is simulated by a two-step finite element method - spectral element method (FEM-SEM) approach [20]. First, the static state of the cable under its own weight is analyzed by means of the FEM. Based on this initial state, the dynamic response of the cable to each hammer impact is analyzed by means of the SEM. The accelerations at the sensor locations are considered as the measured responses. Particularly, each response is polluted by a zero-mean white noise signal, with standard deviation $\sqrt{\sigma}=0.001 \mathrm{~m} / \mathrm{s}^{2}$. Taking the measurement when the sensor array is located at $\mathbf{x}=\{9 \mathrm{~m}, 10 \mathrm{~m}, 11 \mathrm{~m}, 12 \mathrm{~m}\}^{\mathrm{T}}$ for example, the amplitude of the impact force is $A_{\mathrm{h}}=404 \mathrm{~N}$, and the location of the impact is $x_{\mathrm{h}}=31.5 \mathrm{~m}$. The measured acceleration responses are shown in Fig. 21.

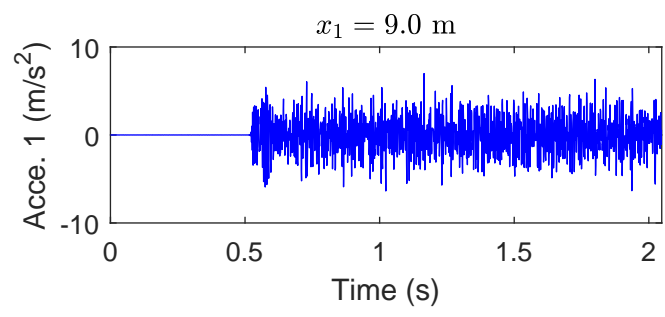

(a)

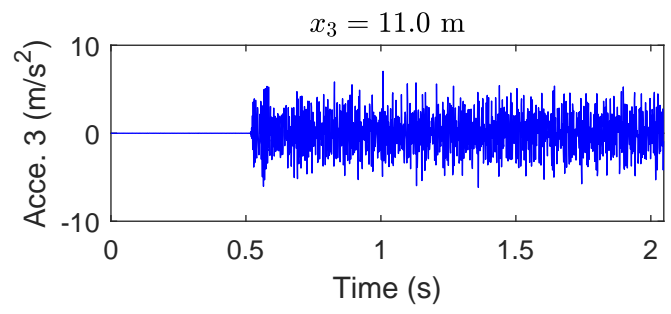

(c)

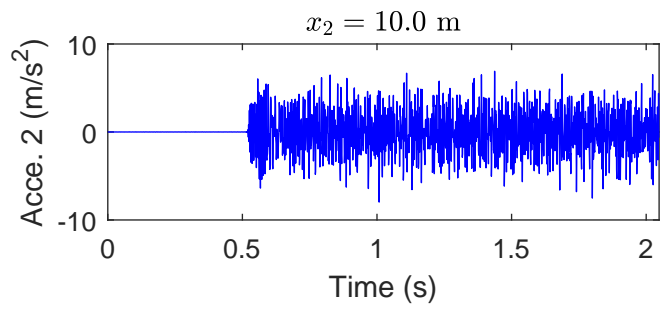

(b)

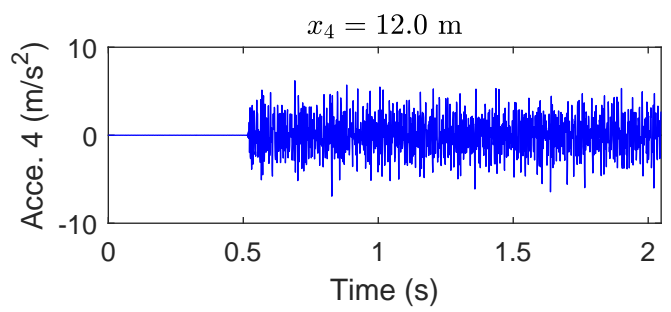

(d)

Figure 21: The measured acceleration responses: a) $\left.\left.\left.x_{1}=9.0 \mathrm{~m} \mathrm{~b}\right) x_{2}=10.0 \mathrm{~m} \mathrm{c}\right) x_{3}=11.0 \mathrm{~m} \mathrm{~d}\right) x_{4}=12.0 \mathrm{~m}$

First of all, the measured time-domain acceleration signal is transformed into the frequency domain by the Laplace transform 20, obtaining $\hat{\mathbf{V}}_{\mathrm{m}}$ in Eq. 67]. The real constant of the complex frequency $s$ is $\sigma=2 \pi /\left(N_{\mathrm{s}} \Delta T\right)$ [28]. For example, the Laplace transform of the measured acceleration response shown in Fig. 21 is given in Fig. 22 .

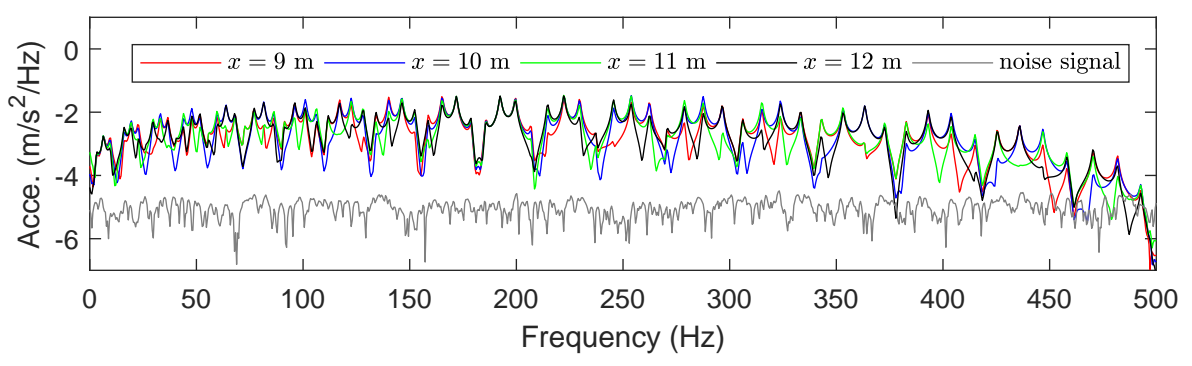

Figure 22: The Laplace transform of the measured response

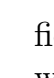$$
1
$$

By substituting the frequency-domain acceleration response $\hat{\mathbf{V}}_{\mathrm{m}}$ (Fig. 22 into Eq. 72 , the wave coefficients $C_{1}^{(\mathrm{es})}, C_{2}^{(\mathrm{es})}, C_{3}^{(\mathrm{es})}$ and $C_{4}^{(\mathrm{es})}$ are estimated. For each hammer impact and sensor array, these four wave coefficients can be estimated. Fig. 23 shows each estimated wave coefficients, as a function of both the location of the sensor array $\mathbf{x}$ and the frequency $\omega$. 


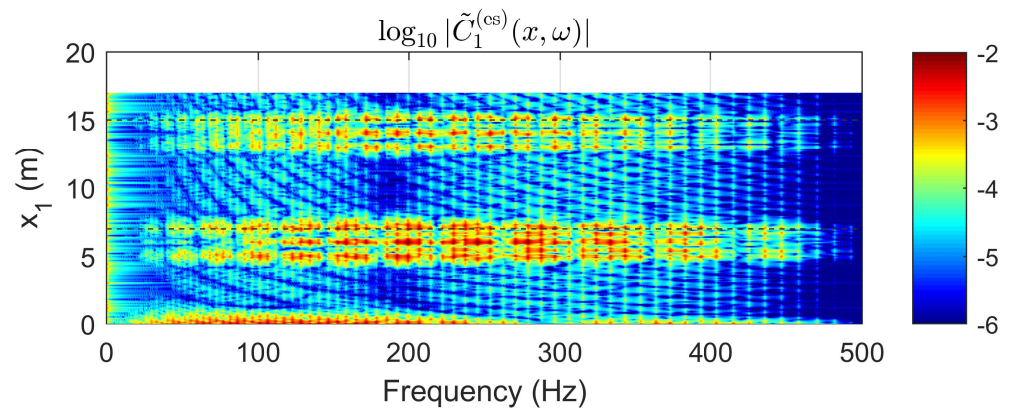

(a)

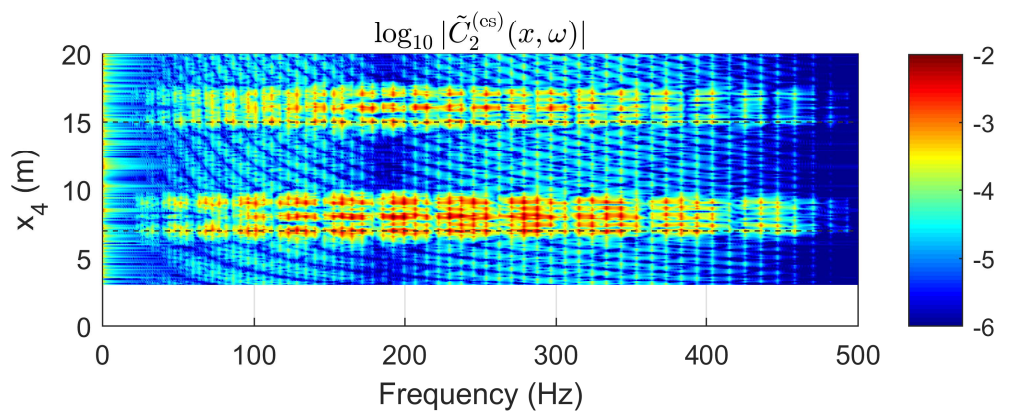

(b)

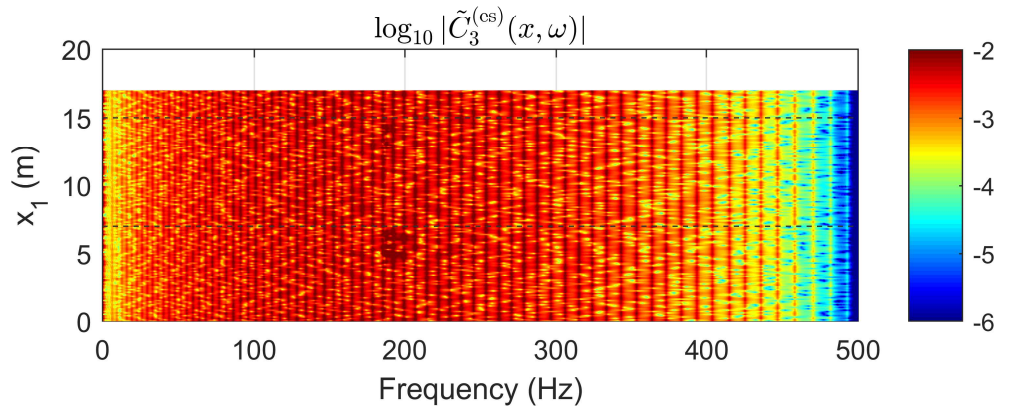

(c)

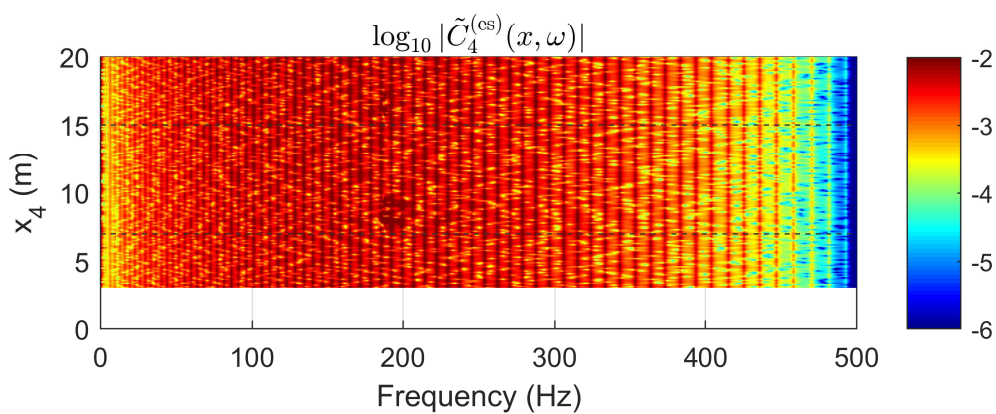

(d)

Figure 23: The estimated wave coefficients by the wave decomposition (The gray dashed lines indicate the damage locations): a) $C_{1}^{(\text {es })}$ b) $C_{2}^{(\text {es })}$ c) $C_{3}^{(\text {es })}$ d) $C_{4}^{(\text {es })}$

In Fig. 23(a) and Fig. 23(b), the evanescent waves are concentrated near the damage locations $(x=7 \mathrm{~m}$ 
and $x=15 \mathrm{~m})$. Additionally in Fig. 23(a) the evanescent wave is detected as well near the left anchor $(x=0 \mathrm{~m})$ due to the discontinuity present. However, the PB wave components (Fig. 23(c) and Fig. 23(d)), which are determined by the global characteristics of the cable, do not reveal the presence of discontinuities. This is because the propagating waves are mainly affected by the amplitude and location of the excitation, which are different for each hammer impact.

Furthermore, by substituting the estimated wave coefficients (Fig. 23) into Eq. (74) and Eq. (75), the REW indicators $\left[r_{12}^{(\mathrm{es})}\right]_{\mathrm{L}}$ and $\left[r_{12}^{(\mathrm{es})}\right]_{\mathrm{R}}$ are estimated (Fig. 24, which depend only on damage level. The physical meaning of the reflection coefficients has been illustrated by Fig. 17

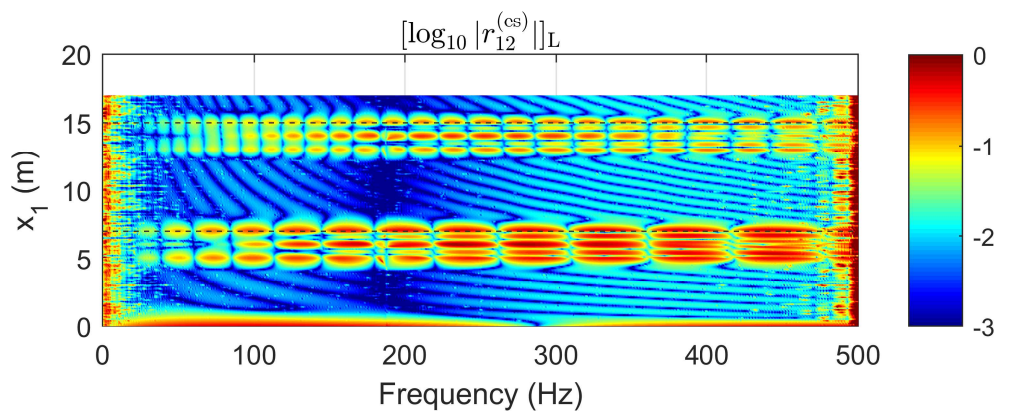

(a)

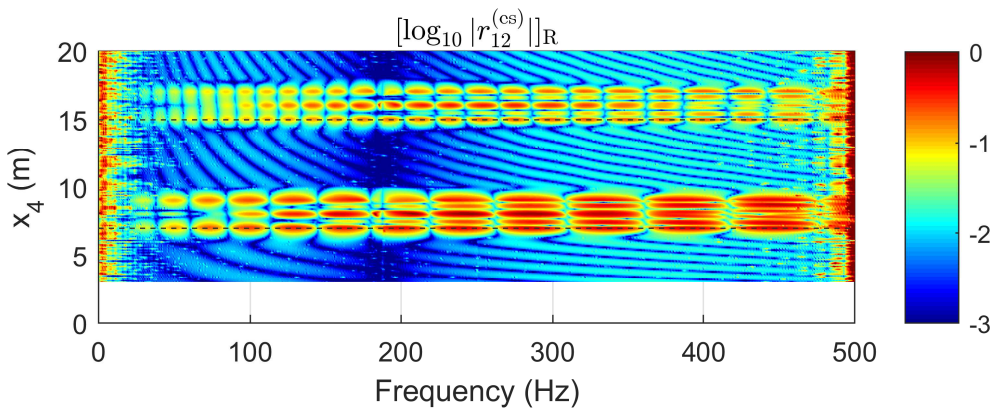

(b)

Figure 24: Estimated REW indicators (The gray dashed lines indicate the damage locations): a) $\left.\left[r_{12}^{(\text {es })}\right]_{\mathrm{L}} \mathrm{b}\right)\left[r_{12}^{(\mathrm{es})}\right]_{\mathrm{R}}$

In Fig. 24, each local damage can be identified independently. The influence of the measurement noise at the frequencies below $10 \mathrm{~Hz}$ and above $450 \mathrm{~Hz}$ is easily recognizable and can for this reason be excluded from the data interpretation. From the results in Fig. 24 it is observed that the evanescent wave components are not strictly concentrated at the damage interface, but instead are smeared over wider areas $(x \in[5,7] \cup[13,15]$ in Fig. 24(a) and $x \in[7,9] \cup[15,17]$ in Fig. 24(b). This phenomenon will be clarified next.

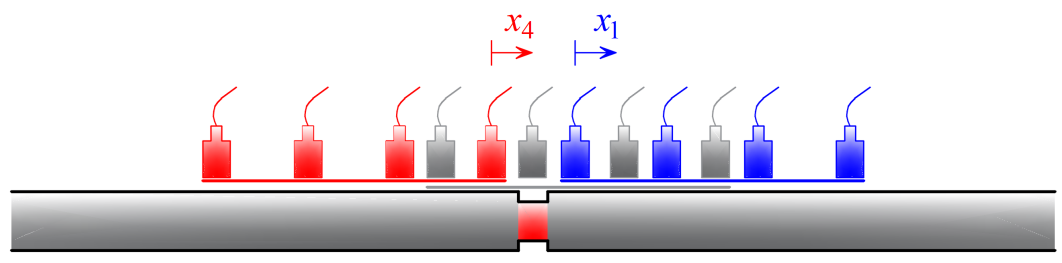

Figure 25: The sensor array passes through the damage location

As shown in Fig. 25, three cases will be considered when the sensor array passes through a damage location: 
1. When the sensor array is to the left of the damage location (the red sensors in Fig. 25), the damage interface is to the right of the substructure, and a significant $\left[r_{12}^{(\mathrm{es})}\right]_{\mathrm{R}}$ is detected.

2. When damage is situated within the substructure (i.e. the gray sensors in Fig. 25), the dynamic response of the substructure does not satisfy Eq. [68). Therefore, Eq. (69) does not hold, and the estimated wave coefficients make no sense, despite the large values of both $\left[r_{12}^{(\mathrm{es})}\right]_{\mathrm{L}}$ and $\left[r_{12}^{(\mathrm{es})}\right]_{\mathrm{R}}$.

3. When the sensor array is to the right of the damage location (the blue sensors in Fig. 25), the damage interface is to the left of the substructure, and a significant $\left[r_{12}^{(\mathrm{es})}\right]_{\mathrm{L}}$ is detected.

In order to indicate the damage location more clearly, an intensity of the evanescent wave reflection is defined by integrating $\left|\left[r_{12}^{(\mathrm{es})}\right]_{\mathrm{L}}\right|$ and $\left|\left[r_{12}^{(\mathrm{es})}\right]_{\mathrm{R}}\right|$ along the frequency axis, with regard to the frequency range $\omega \in\left[\omega_{1}, \omega_{2}\right]$ where the signal-to-noise ratio is considered sufficiently high:

$$
\begin{aligned}
& E_{\mathrm{L}}(x)=\frac{1}{\left|\omega_{2}-\omega_{1}\right|} \int_{\omega_{1}}^{\omega_{2}}\left|\left[r_{12}^{(\mathrm{es})}\right]_{\mathrm{L}}(x, \omega)\right| \mathrm{d} \omega \\
& E_{\mathrm{R}}(x)=\frac{1}{\left|\omega_{2}-\omega_{1}\right|} \int_{\omega_{1}}^{\omega_{2}}\left|\left[r_{12}^{(\mathrm{es})}\right]_{\mathrm{R}}(x, \omega)\right| \mathrm{d} \omega
\end{aligned}
$$

where $E_{\mathrm{L}}(x)$ and $E_{\mathrm{R}}(x)$ indicate the intensity of the evanescent wave reflection from the left side and the right side of the substructure, respectively. At a certain location, the sum of the above two terms:

$$
E_{\mathrm{sum}}(x)=E_{\mathrm{L}}(x)+E_{\mathrm{R}}\left(x-L_{\mathrm{s}}\right)
$$

represents the total intensity of the evanescent wave reflections to both sides, which can be used as an indicator for damage localization.

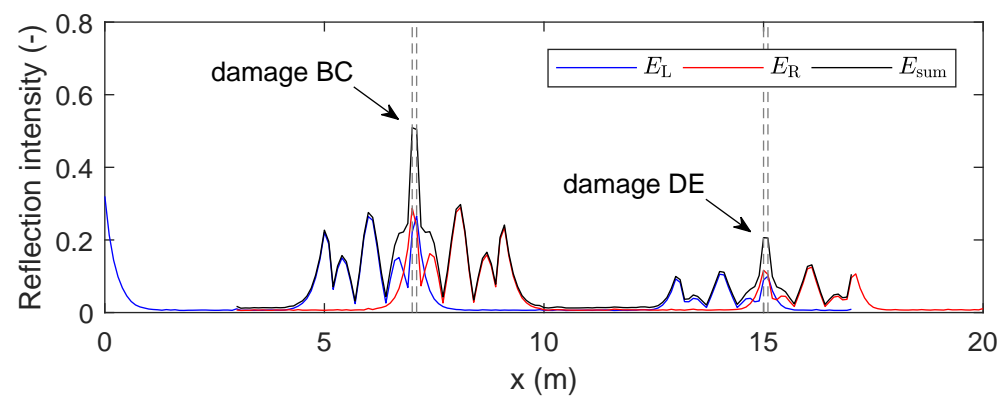

Figure 26: Damage localization based on the intensity of the evanescent wave reflection (The gray dashed lines indicate the damage interfaces)

Fig. 26 shows the intensity of the evanescent wave reflection of the cable, considering $\omega_{1}=10 \mathrm{~Hz}$ and $\omega_{2}=450 \mathrm{~Hz}$ to exclude the effect of the noise. From the peaks of $E_{\text {sum }}$, the damage locations $(x=7 \mathrm{~m}$ and $x=15 \mathrm{~m}$ ) are identified. Based on the known damage locations, the estimated REW at the locations $0.1 \mathrm{~m}$ away from the damage interfaces (i.e., $\left|\left[r_{12}^{(\mathrm{es})}\right]_{\mathrm{R}}(x=6.9 \mathrm{~m}, \omega)\right|$ and $\left|\left[r_{12}^{(\mathrm{es})}\right]_{\mathrm{L}}(x=7.2 \mathrm{~m}, \omega)\right|$ for the damage BC, $\left|\left[r_{12}^{(\mathrm{es})}\right]_{\mathrm{R}}(x=14.9 \mathrm{~m}, \omega)\right|$ and $\left|\left[r_{12}^{(\mathrm{es})}\right]_{\mathrm{L}}(x=15.2 \mathrm{~m}, \omega)\right|$ for the damage DE) are extracted for the quantification of the damage levels. The theoretical value of the REW $\left(r_{12}\right)$ can be evaluated by Eq. (28). Based on this, the reference value of the REW at $x_{\mathrm{s}}$ away from the damage interface is then calculated by:

$$
r_{12}^{*}=r_{12} \cdot \exp \left(-k_{2} x_{\mathrm{s}}\right)
$$

In this example, $x_{\mathrm{s}}=0.1 \mathrm{~m}$. The reference values and the estimated values of the REW are compared in Fig. 27. 


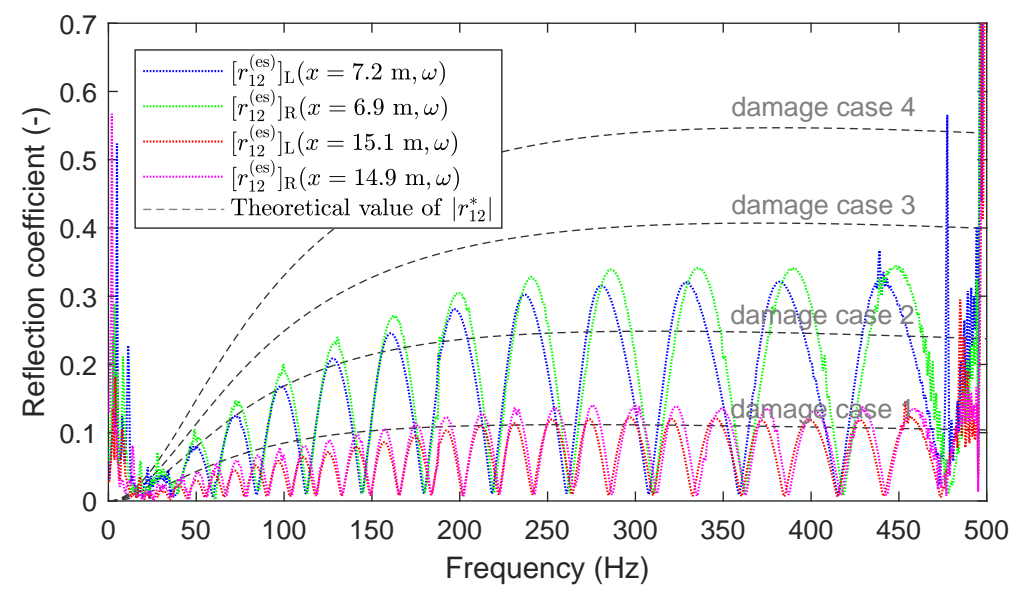

Figure 27: Damage quantification based on the estimated REW indicator

From the comparison between the estimated REW and the theoretical value of $\left|r_{12}^{*}\right|$, shown in Fig. 27, the damage levels at $\mathrm{BC}$ and $\mathrm{DE}$ are identified, corresponding to damage case 2 and 1 in Tab. 1 respectively. At frequencies below $10 \mathrm{~Hz}$ and above $450 \mathrm{~Hz}$, the estimated REW is apparently affected by the measurement noise, and these results can be easily disregarded. The following two issues should be noticed for the results shown in Fig. 27.

1. The estimated REW do not perfectly match the reference values at each frequency. The envelope of the frequency-domain REW approaches the theoretical value, which gives an approximate estimation of the damage level.

2. The damage levels are always overestimated by the proposed indicator, as the estimated values of REW indicator $\mid\left[r_{12}^{(\mathrm{es})}\right]$ are slightly higher than the reference values. This is even more obvious in the case of a higher damage level.

The reason is that the cable model is not exactly consistent with the reference model (the single damage interface in Fig. 2), instead, each local damage involves two interfaces. As shown in Fig. 28, the reflection of the evanescent wave at the damage interface $\left(r_{12}\right)$ is indeed correct. However, the transmitted evanescent wave $\left(t_{12}\right)$ is further transmitted outside the damage part through the other interface ( $\delta$ in Fig. 28). The reflection of the evanescent wave is therefore interfered by such additional component, resulting in the overestimation and interference pattern of Fig. 27

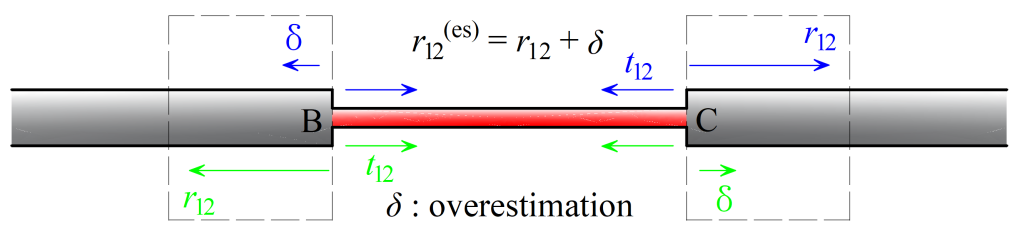

Figure 28: The transmission of evanescent wave through the damage part

Despite the influence of the transmitted wave, the damage level can still be assessed by the proposed indicator, with only a slight overestimation. From the above study, it can be seen that the proposed indicator is affected by neither the global response of the cable nor the differences in the amplitudes and locations of individual hammer impacts. Additionally, the damage indicator REW is frequency dependent, providing multiple values. These values at different frequencies can be combined into a single indicator which is more robust with respect to measurement noise. 


\section{Conclusion}

In the present work, the frequency-domain response of a cable is decomposed into evanescent wave and propagating bending $(\mathrm{PB})$ wave components. Based on this, a wave-based methodology involving the reflection of the evanescent wave is proposed for local damage identification.

First, it is proven by an analytical study that the evanescent wave is locally sensitive to discontinuities in the bending stiffness of the cable. In turn, a signal processing method is proposed to decompose the frequency-domain response of cable into evanescent wave and PB wave components. This allows the estimation of the REW for incident PB wave, which depends only on the characteristics of discontinuity and is proposed as a local damage indicator. The total intensity of REW can be used for localization of the damage. Based on the known damage location, local damage can be quantified by taking the theoretical value of $r_{12}^{*}$ as a reference.

The feasibility of the proposed methodology is studied by means of a numerical experiment simulated by a two-step FEM-SEM approach. The advantages of the proposed methodology are:

1. The evanescent wave is always concentrated near a discontinuity, and decays exponentially with distance. For this reason, multiple local damages can be identified independently.

2. For the substructure defined by the location of the sensor array, the dynamic response is induced by the motion at both end points, satisfying the analytical solution. Physical excitation is therefore not particularly restricted as long as the excitation is neither inside the substructure nor near the sensors.

3. The estimated REW only depends on the characteristics of discontinuity. The spectra of the global response do not affect the estimated result as long as the concerned frequency band is sufficiently excited.

4. The estimation of wave components is obtained by a linear transform of the measured response, from frequency domain to wavenumber domain. The frequency-dependent REW therefore provides a series of independent values, which can be used to derive a damage indicator which is more robust to the measurement noise.

The proposed methodology is promising for application in cable structures, offering opportunities to vibrationbased damage assessment and structural health monitoring. The methodology can be extended for application to beams, either with or without prestressing, by considering $N_{\mathrm{x}}=0$ or $N_{\mathrm{x}}<0$, respectively.

\section{Acknowledgment}

The first author is an international scholar in the Department of Civil Engineering, KU Leuven, supported by the China Scholarship Council (CSC) (201607000039). The financial support is gratefully acknowledged.

\section{References}

[1] H. M. Irvine, Cable structures, New York: ASCE Press, 1992.

$[2]$ L. S. Frank, P. G. Christopher, Cable corrosion in bridges and other structures: Causes and solutions, New York: ASCE Press, 1996.

[3] J. M. Stallings, K. H. Frank, Stay-cable fatigue behavior, Journal of Structural Engineering 117 (3) (1991) $936-950$.

[4] H. Svensson, Cable-stayed bridges: 40 years of experience worldwide, John Wiley \& Sons, 2013.

[5] A. B. Mehrabi, N. M. Telang, Cable-stayed bridge performance evaluationlessons from the field, in: Proceedings of the Structures Congress and Exposition, Vol. 1083, 2003.

[6] H. N. Ho, K. D. Kim, Y. S. Park, J. J. Lee, An efficient image-based damage detection for cable surface in cable-stayed bridges, NDT \& E International 58 (3) (2013) 18-23.

[7] A. B. Mehrabi, In-service evaluation of cable-stayed bridges, overview of available methods and findings, Journal of Bridge Engineering 11 (6) (2006) 716-724.

[8] X. Liu, J. Xiao, B. Wu, C. He, A novel sensor to measure the biased pulse magnetic response in steel stay cable for the detection of surface and internal flaws, Sensors \& Actuators A Physical 269 (2018) 218-226.

[9] B. Peeters, G. De Roeck, Stochastic system identification for operational modal analysis: a review, Journal of Dynamic Systems, Measurement, and Control 123 (4) (2001) 659-667.

[10] O. S. Salawu, Detection of structural damage through changes in frequency: a review, Engineering Structures 19 (9) (1997) $718-723$. 
[11] A. K. Pandey, M. Biswas, M. M. Samman, Damage detection from changes in curvature mode shapes, Journal of Sound and Vibration 145 (2) (1991) 321-332.

[12] S. W. Doebling, C. R. Farrar, M. B. Prime, et al., A summary review of vibration-based damage identification methods, Shock and Vibration Digest 30 (2) (1998) 91-105.

[13] G. De Roeck, The state-of-the-art of damage detection by vibration monitoring: the simces experience, Structural Control and Health Monitoring 10 (2) (2003) 127-134.

[14] D. Degrauwe, G. De Roeck, G. Lombaert, Uncertainty quantification in the damage assessment of a cable-stayed bridge by means of fuzzy numbers, Computers \& Structures 87 (1718) (2009) 1077-1084.

[15] Y. Shokrani, V. K. Dertimanis, E. N. Chatzi, M. N. Savoia, On the use of mode shape curvatures for damage localization under varying environmental conditions, Structural Control \& Health Monitoring (3) (2018) e2132.

[16] N. Stubbs, J. T. Kim, K. Topole, An efficient and robust algorithm for damage localization in offshore platforms, in: Proceedings of the ASCE 10th Structures Congress, Vol. 1, 1992, pp. 543-546.

[17] Z. Y. Shi, S. S. Law, L. M. Zhang, Structural damage localization from modal strain energy change, Journal of Sound and Vibration 218 (5) (1998) 825-844.

[18] D. Anastasopoulos, M. De Smedt, L. Vandewalle, G. De Roeck, E. Reynders, Damage identification using modal strains identified from operational fiber-optic bragg grating data, Structural Health Monitoring (2017) 1475921717744480.

[19] J. F. Doyle, Wave propagation in structures - spectral analysis using fast discrete Fourier transforms.

[20] S. H. Zhang, R. L. Shen, T. Wang, G. De Roeck, G. Lombaert, A two-step FEM-SEM approach for wave propagation analysis in cable structures, Journal of Sound and Vibration 415 (2018) 41-58.

[21] M. J. S. Lowe, D. N. Alleyne, P. Cawley, Defect detection in pipes using guided waves, Ultrasonics 36 (1-5) (1998) 147-154.

[22] J. L. Rose, M. J. Avioli, P. Mudge, R. Sanderson, Guided wave inspection potential of defects in rail, NDT \& E International 37 (2) (2004) 153-161.

[23] C. Schaal, S. Bischoff, L. Gaul, Damage detection in multi-wire cables using guided ultrasonic waves, Structural Health Monitoring 15 (3) (2016) 279-288.

[24] P. S. Lowe, R. Sanderson, S. K. Pedram, N. V. Boulgouris, P. Mudge, Inspection of pipelines using the first longitudinal guided wave mode, Physics Procedia 70 (2015) 338-342.

[25] C. Schaal, S. Bischoff, L. Gaul, Energy-based models for guided ultrasonic wave propagation in multi-wire cables, International Journal of Solids and Structures 64 (2015) 22-29.

[26] K. Spak, G. Agnes, D. Inman, Parameters for modeling stranded cables as structural beams, Experimental Mechanics 54 (9) (2014) 1613-1626.

[27] M. K. Yücel, M. Legg, V. Kappatos, T. H. Gan, An ultrasonic guided wave approach for the inspection of overhead transmission line cables, Applied Acoustics 122 (2017) 23-34.

[28] D. J. Wilcox, Numerical Laplace transformation and inversion, International Journal of Electrical Engineering Education 15 (3) (1978) 247-265.

[29] C. Mei, B. R. Mace, Wave reflection and transmission in Timoshenko beams and wave analysis of timoshenko beam structures, Journal of Vibration and Acoustics 127 (4) (2005) 382-394.

[30] C. H. Wang, L. R. F. Rose, Wave reflection and transmission in beams containing delamination and inhomogeneity, Journal of Sound and Vibration 264 (4) (2003) 851-872. 